\title{
Old-Age Care Prevalence in Switzerland: Drivers and Future Development*
}

\author{
Michel Fuino, Joël Wagner ${ }^{\dagger}$
}

\begin{abstract}
Long-term care (LTC) delivered to elderly persons in need of assistance in activities of daily living is a topic of increasing importance. The financing of LTC, the needs for specialized infrastructure and the limited number of caregivers will pose a systemic threat in many developed countries. In this paper, we analyze the factors influencing the old-age care prevalence rates in Switzerland through a log-linear regression model. Based on a cross-sectional dataset covering the LTC needs from 1995 to 2014, we statistically support the effect of key drivers such as the age, the gender and the region of residence. We distinguish the prevalence by the mild, moderate and severe frailty levels and by care received either at home or in an institution. Our regression results evidence that prevalence rates exponentially increase with the age yielding significantly higher values for women. These effects are emphasized for moderate and severe dependence and for institutional care. Finally, we forecast the number of dependent persons until 2045. Our projections reveal an important increase in the future numbers. While we observe that the dependent population more than doubles over the 30-year horizon, we report significant cantonal differences. Our results are relevant to governments, practitioners and academics alike and help to better understand the factors affecting the demand of LTC and predicting future needs.
\end{abstract}

Key words long-term care $\cdot \log$-linear regression $\cdot$ prevalence rates $\cdot$ forecast

\section{Introduction}

Population aging is a topic affecting most of the developed countries. While the increase in life expectancy at birth drives the overall development (United Nations, 2016), the growing demand for long-term care (LTC) delivered to elderly persons in need of assistance with activities of daily living (ADL) creates a systemic threat for the society. First, the financing of LTC depends upon the development of sustainable schemes in order to alleviate the share paid by households (Colombo, 2012; Rockinger and Wagner, 2016). Second, the limited space in existing infrastructures offering LTC will require reorganizing the care system and eventually new constructions (Katz, 2011; Cosandey, 2016; El Bernoussi and Rockinger, 2017). Finally, the future needs of care are likely to come along with a lack of qualified staff (Nichols et al., 2010; World Health Organization, 2014). Switzerland is the country with the highest out-of-pocket spending (Weaver, 2012; Swiss Re, 2014). This financial burden left on households reveals the absence of appropriate social and private insurance cover. The main problem lies in individuals

\footnotetext{
${ }^{*}$ This is a post-peer-review, pre-copyedit version of an article published in European Actuarial Journal. The final authenticated version is available online at: https://doi.org/10.1007/s13385-018-0185-3.

${ }^{\dagger}$ Michel Fuino (michel.fuino@unil.ch) and Joël Wagner (joel.wagner@unil.ch) are with the Department of Actuarial Science, Faculty of Business and Economics, University of Lausanne. The second author is also with the Swiss Finance Institute, University of Lausanne, Switzerland. Financial support from the Swiss National Science Foundation (grant no. 100018_169662) and the Swiss Insurance Association is gratefully acknowledged.
} 
underestimating the cost of LTC or being unaware that such care is not paid by their health insurance (Pauly, 1990). They neither save money nor take an insurance for covering their possible future LTC need. Consequently, the state has to provide allowances for mitigating this financial burden. Such social participation in the costs creates a crowding out-effect for private insurance solutions (Brown et al., 2007; Le Corre, 2012). Thereafter, we observe the substitution of formal LTC through help provided by relatives (Bonsang, 2009; Courbage et al., 2018). This is also partially due to intergenerational moral hazard by parents willing to be cared for by their children (Courbage and Zweifel, 2011).

The contribution of the present work is twofold. First, we identify the main drivers for the demand and the development of LTC in Switzerland on the period from 1995 to 2014. We base our approach on prevalence rates defined by the ratio of elderly in need of LTC at a given age over the total population at the same age. Using a log-linear regression framework, we investigate on different models explaining the dependence between the drivers and the needs for LTC at different frailty levels and types of care. Second, building on the previously developed models, the historic data and the national demographic forecast, we project the future number of elderly in need of care with ADLs for years up to 2045.

A large body of literature has raised the relevance of sociodemographic factors when studying dependence in LTC. In their work, De Meijer et al. (2011) find that an individual's age, gender and cause of death significantly affect the demand for LTC. However, the precise effect is not well understood. For example, when controlling for the time to death, some variables like the age lose importance in shaping the LTC need (Zweifel et al., 2004). Furthermore, the application of multistate models for explaining the evolution of dependency requires to consider frailty levels and types of care (Czado and Rudolph, 2002) as well as the time spent in dependency (Fuino and Wagner, 2018). Some authors have considered the importance of culture in determining the demand for care in Switzerland. Eugster et al. (2011) find that residents of Latin-speaking regions receive social health insurance benefits more frequently than residents of the German-speaking region. On the opposite, the demand for LTC is higher in the Germanspeaking region (Gentili et al., 2017).

The link between LTC demand and the drivers can be studied through different models. Loglinear ordinary least squares regression models are widely used in empirical economics studies (Wooldridge, 2013). Such models allow for accurate estimates in the presence of an exponential relation with the independent variables and usually offer an appropriate correction for right-skewed distributions and for the presence of heteroscedasticity (Manning and Mullahy, 2001; Ai and Norton, 2008). Note that a broad range of alternative estimation techniques are available. For example, Black and Craig (2002) use Bayesian inference for estimating disease prevalence rates in the case of data collected from diagnostic tests. Recent literature is also concerned with forecasting the future LTC needs. Fong et al. (2017) and Fong (2017) project future disability trends for elderly individuals basing their approach on time-series methods. Kwon et al. (2012) predict future LTC needs in Korea basing their forecast on a multi-state model. In Eggink et al. (2016), the authors include sociodemographic characteristics (e.g., age, sex, education level) and health status (physical disabilities, chronic disorders and dementia) in their study for the Netherlands. In comparison, Karlsson et al. (2006) take into account the types of care (nursing home, residential home and informal care) and the frailty levels (no disability, moderate disability and severe disability) when forecasting LTC needs in the UK. Finally, Costa- 
Font et al. (2008) lay out how the choice of a demographic scenario can influence the predictions.

In this paper, our aim is to provide analyses on key drivers determining LTC prevalence rates in Switzerland. In the first part, using log-linear regression models, we study the effect on the prevalence rates of six characteristics which are the individual's age, gender, and canton of residence, the calendar year, the frailty level (mild, moderate and severe) and the type of care received (at home or in an institution). Our work is based on a unique cross-sectional dataset covering the total dependent population aged over 65 years in Switzerland during the 1995-2014 20-year period. In the second part, based on the developed models, the data and the available population forecast, we derive predictions of the prevalence rates for the years from 2015 to 2045. A comparison of the expected development along the cantons of Switzerland, the frailty levels and the types of care is presented. Our main results include statistical estimates for the various drivers' effect on prevalence rates and a forecast of the future dependent population until 2045. We find that the individual's age, gender, canton of residence as well as the frailty level and the type of care significantly affect the prevalence rates. They are higher for older persons and for females. Furthermore, they increase with the acuity of the dependence and with the institutional type of care, i.e., we find higher prevalence rates for elderly in severe dependence and being cared for in an institution. Finally, our forecasts show the importance of distinguishing cantons of residence, frailty levels and types of care. With regard to the cantons, we observe three clusters. For the cantons with the highest number of dependent persons, we predict the demand for LTC to be multiplied by a factor of 2.45 until 2045. The two other clusters concern cantons with relatively higher and lower predicted increases in the future dependent population. In the discussion of the findings, we link this to their differing demographic structure and urbanization level.

The remainder of this article is organized as follows: Section 2 introduces the LTC system in Switzerland and presents the distinction of the frailty levels and the types of care in dependence. We briefly lay out some LTC statistics and key drivers observed in the last 20 years. In Section 3, we develop the regression models on which we base our analysis. We present the available data, apply the models and discuss the results including robustness analysis in Section 4. In Section 5, we propose and implement a prediction model for the future population in need of care by canton, by frailty level and by type of care. We conclude in Section 6 .

\section{Overview of the long-term care system in Switzerland}

In this section, we study the Swiss LTC system. We first consider the evolution of the prevalence rates and the main factors of influence. Then, we introduce the available data and describe the major characteristics of the underlying population. A discussion on the demographic forecast of the elderly population up to the year 2045 concludes this section.

\subsection{Description of LTC financing in the Swiss system}

Switzerland is one of the countries with the highest life expectancy at birth (82.9 years in 2014, OECD, 2015). It is composed by 26 cantons and three linguistic regions ${ }^{1}$ and has a population of

\footnotetext{
${ }^{1}$ Three linguistic regions are distinguished in Switzerland. These regions are (1) the German-speaking region comprising the cantons of Aargau (AG), Appenzell Innerrhoden (AI), Appenzell Ausserrhoden (AR), Bern (BE), Basel-Landschaft (BL), Basel-Stadt (BS), Glarus (GL), Graubünden (GR), Lucerne (LU), Nid-
} 
around 8.2 million inhabitants from which more than 1.5 million are older than 65 years (Federal Statistic Office, FSO, 2015). Since the proportion of elderly is expected to be increasing (see Section 2.4), the understanding of LTC, i.e., the care needed for persons suffering from limitations in activities of daily living (ADL), is important. The Swiss Old Age Social Insurance (OASI) law defines six ADLs, namely getting in or out of bed, dressing, bathing, eating, going to the toilet and moving indoors and outdoors (Federal Assembly, 2017). Based on this measure, the acuity of the dependence is classified under three levels. The mild level concerns persons in need of regular assistance for at least two ADLs or permanent personal supervision. The moderate level characterizes dependents in need of assistance for at least two ADLs and permanent personal supervision. The severe level defines elderly persons needing regular assistance with all the ADLs and in addition entails permanent personal supervision. Furthermore, the OASI law differentiates between care received at home and care received in an institution. Care delivered at home concerns individuals receiving care in their own residence without accommodation services while care received in an institution includes accommodation.

The financing of LTC involves three paying agents. First, the first pillar of the Swiss old-age provisions system provides non means-tested benefits depending both on the acuity level and the canton of residence. Although if the amount of the allowance differs by cantons, the assessment rules are defined at the federal level and apply identically to the whole country. Second, the compulsory health insurance pays for medication to avoid health deterioration and for ad hoc care provided by professionals. Third, the accommodation related costs (e.g., laundry, feeding) are left to the private households. Since accommodation services generate high costs, it follows that institutional care are significantly more expensive than care at home. Therefore, the type of care received influences the out-of-pocket amount to be paid by the individual itself.

\subsection{Evolution of the LTC needs over the period from 1995 to 2014}

In Switzerland, two offices produce statistics on old-age care and population dynamics. On the one hand, the Swiss Central Compensation Office $(\mathrm{CCO})^{2}$ specializes in the benefits paid under the old-age insurance scheme concerning both pension and disability benefits. With regard to LTC, the CCO can provide information on the gender, the age, the canton of residence, the acuity level (mild, moderate or severe) and the type of care (at home or in an institution) of dependent elderly receiving old-age care benefits. The longitudinal dataset we could obtain from the CCO covers a 20-year period from 1995 to 2014 (see also Section 4.1). On the other hand, the Swiss Federal Statistical Office $(\mathrm{FSO})^{3}$ provides aggregated statistics on the population, the demographics and the migration. From this second source, we get access to the historical census of the population (1995-2014) allowing to calculate prevalence rates and to the population forecast scenarios (2015-2045) laying the basis for our projections.

Table 1 reports descriptive statistics for the period from 1995 to 2014 on the population registered for old-age care benefits, the total population aged $65+$ (i.e., 65 years or older) in Switzerland and the corresponding LTC prevalence rates. The row labeled "CCO data" reports

walden (NW), Obwalden (OW), St.Gallen (SG), Schaffhausen (SH), Solothurn (SO), Schwyz (SZ), Thurgau (TG), Uri (UR), Zug (ZG), and Zurich (ZH); (2) the French-speaking region comprising the cantons of Fribourg (FR), Geneva (GE), Jura (JU), Neuchâtel (NE), Vaud (VD), and Valais (VS); and (3) the Italian-speaking region formed by the canton of Ticino (TI).

${ }^{2}$ www.zas.admin.ch

${ }^{3}$ www. bfs . admin.ch 
the aggregated and corrected number of elderly persons derived from the $\mathrm{CCO}$ data at the end of each year, while the "FSO data" row reports publicly available figures published by the FSO in its aggregate statistics starting only from the year 1999. The cross-sectional view constructed from the CCO micro data overestimates the FSO numbers on average by $10.71 \%{ }^{4}$ Therefore, the $\mathrm{CCO}$ data and the prevalence rates reported in the table are corrected by this factor to more closely reflect the published statistics. This does not affect the distribution figures reported. From our statistics, we observe that the population registered for old-age care benefits increases through the years. In 1995, we count about 33.5 thousand individuals. This number has gradually increased and reaches 58.9 thousand in 2014 .

Looking at the distribution of the population registered for old-age care benefits between the 65-79 and the 80+ age groups, we observe stable proportions over the years, with about $65 \%$ being over 80 -years old. The breakdown among both genders reveals a higher proportion of female (women represent about two thirds of the registered individuals in 2014). Women in dependency have a mean age between 83.6 and 84.0 years which is 3.5 to 5 years higher than the men's one. The gender differences in the prevalence and in the mean age can be explained by the longer life expectancy of dependent females (Mathers et al., 2001; Schünemann et al., 2017; Fong et al., 2017). Table 1 also reports the distribution by linguistic regions, frailty levels and types of care. The distribution of the dependent population in the three linguistic regions closely follows the distribution of the total population in Switzerland, where about $65 \%$ of the population lives in the German-speaking region, $27 \%$ in the French-speaking region and $8 \%$ in the Italian-speaking region. ${ }^{5}$ The statistics on the distribution by frailty levels and by types of care significantly change through the three sub-periods from 1995 to 2000, from 2001 to 2010 and from 2011 to 2014. For the period from 1995 to 2000, no benefits are offered to elderly being cared for at home because they are only recognized since 2001. Further we observe an important increase in both the share of mildly dependent persons and of elderly cared for at home when comparing the two periods 2001-2010 and 2011-2014. Indeed, benefits for mildly dependent persons living at home are paid only since 2011.

The bottom of Table 1 reports figures on the total population of elderly persons in Switzerland and the prevalence rates. For 1995, the Swiss population census records 1044 thousand elderly aged over 65 years. They are 1465 thousand in 2014. We calculate the prevalence rates by dividing the number of dependent persons reported by the CCO by the total population. Starting with a value of $3.2 \%$ in 1995, the prevalence rates persist at the same level until 2011. After 2011, the prevalence rates remain stable at $4.0 \%$. The evolution of prevalence rates through the years is illustrated and discussed on the hand of the Figure 1b below.

The graphs in Figure 1 illustrate the evolution of the population aged $65+$ and of the prevalence rates from 1995 to 2014. Using an index, Figure 1a relates the increase in the total population aged $65+$ and the variation in the population registered for old-age care over the considered 20-year time frame. The population in 1995 defines the baseline of the index (1.00) for both curves. For example, in 2005, the value of the index is approximately 1.18 (increase of $18 \%$ in ten years) for the $65+$ population and 1.29 (increase of 29\%) for the sub-population registered

\footnotetext{
${ }^{4}$ Differences in the numbers may arise from the exact registration dates of the acuity levels, how up-to-date the sources are, if former years are revised over time, the processes for aggregation used, and the cleaning of incomplete entries.

${ }^{5}$ www.bfs.admin.ch/bfs/en/home/statistics/population.html
} 


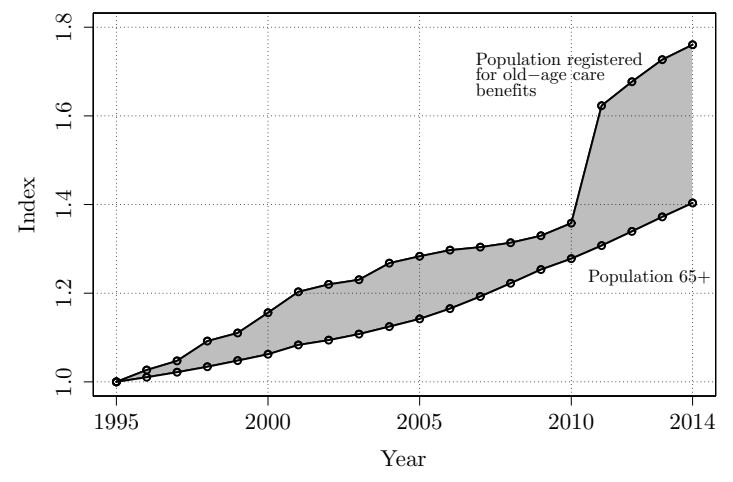

(a) Index

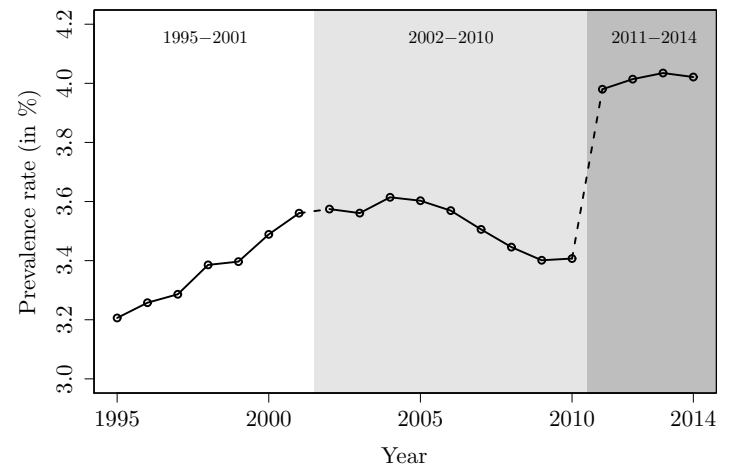

(b) Prevalence rates

Figure 1: Population registered for old-age care benefits for the years from 1995 to 2014.

for old-age care benefits. Figure $1 \mathrm{~b}$ reports the prevalence rates over time and distinguishes the three sub-periods 1995-2000, 2001-2010 and 2011-2014. Both graphs (a and b) show an important change in the population registered for old-age care benefits in 2011. The increase in the population registered for old-age care and thus of the prevalence rate is a consequence stemming from the implementation of the new LTC financing scheme (Weaver, 2012). This new scheme partly implies the incentivization of care delivered at home versus institutional care by recognizing the role of informal care through the attribution of a specific allowance to the relatives. This is particularly observable in the increase in the share of dependent persons with mild severity living at home (cf. Table 1).

\subsection{Key drivers of LTC prevalence}

The LTC prevalence rates discussed above depend on sociodemographic factors like the individual's gender, age and canton of residence. Furthermore, they can be split by frailty level and type of care. The graphs in Figure 2 report the prevalence rates by age for the three frailty levels (mild, moderate and severe, see Fig. 2a) and for the two types of care (at home and in an institution, see Fig. 2b) in 2014. Both graphs show prevalence rates increasing with the age. The first graph illustrates the similar growth of prevalence rates for severely and moderately dependent individuals. The increase in prevalence rates for mildly dependent persons is significantly smaller with only a three percent point difference in prevalence rates between 65-years and 95-years old persons. In the second graph, we distinguish two patterns for persons aged 80 years and older. The first pattern depicts an important increase in the prevalence rate for persons being cared for in an institution. After an age of 95 years, they represent about 25\% of the elderly population. The second pattern identifies a moderate increase for people being cared for at home.

Figure 3 presents the prevalence rates in 2014 by gender and by age (Fig. 3a) and by canton (Fig. 3b). In the first figure, the solid line reports the total prevalence in 2014 while the shaded area is bounded by the minimum and maximum values of the prevalence rates between 1995 and 2014. Comparing the rates for males and females in 2014, we observe that female aged over 80 years show significantly higher prevalence rates than male. This difference in prevalence rates exceeds five percent point when looking into males and females in their nineties. In the second 


\begin{tabular}{|c|c|c|c|c|c|c|c|c|c|c|c|c|c|c|c|c|c|c|c|c|c|}
\hline Year & & 1995 & 1996 & 1997 & 1998 & 1999 & 2000 & 2001 & 2002 & 2003 & 2004 & 2005 & 2006 & 2007 & 2008 & 2009 & 2010 & 2011 & 2012 & 2013 & 2014 \\
\hline \multicolumn{22}{|c|}{ Population registered for old-age care benefits } \\
\hline $\mathrm{CCO}$ data $\mathrm{t}$ & th. & 33.5 & 34.4 & 35.1 & 36.6 & 37.2 & 38.7 & 40.3 & 40.8 & 41.2 & 42.4 & 43.0 & 43.4 & 43.7 & 44.0 & 44.5 & 45.5 & 54.3 & 56.0 & 57.8 & 58.9 \\
\hline FSO data $\mathrm{t}$ & th. & n.a. & n.a. & n.a. & n.a. & 37.8 & 38.4 & 39.2 & 41.4 & 42.6 & 42.6 & 44.1 & 44.6 & 45.6 & 45.7 & 45.8 & 44.5 & 51.1 & 53.8 & 55.8 & 57.4 \\
\hline \multicolumn{22}{|c|}{ Distribution by age groups } \\
\hline 65-79 & $\%$ & 35.0 & 35.6 & 36.3 & 36.2 & 36.5 & 36.2 & 35.4 & 35.3 & 34.9 & 34.4 & 34.4 & 34.3 & 34.5 & 34.6 & 35.0 & 34.9 & 34.4 & 34.7 & 35.0 & 35.3 \\
\hline $80+\quad 9$ & $\%$ & 65.0 & 64.4 & 63.7 & 63.8 & 63.5 & 63.8 & 64.6 & 64.7 & 65.1 & 65.6 & 65.6 & 65.7 & 65.5 & 65.4 & 65.0 & 65.1 & 65.6 & 65.3 & 65.0 & 64.7 \\
\hline \multicolumn{22}{|c|}{ Distribution by gender } \\
\hline Male $\quad \%$ & $\%$ & 29.0 & 29.1 & 29.4 & 29.6 & 29.8 & 30.1 & 30.2 & 30.2 & 30.3 & 30.3 & 30.5 & 30.6 & 31.0 & 31.3 & 31.8 & 32.2 & 32.5 & 33.0 & 33.3 & 33.5 \\
\hline Female $\quad ?$ & $\%$ & 71.0 & 70.9 & 70.6 & 70.4 & 70.2 & 69.9 & 69.8 & 69.8 & 69.7 & 69.7 & 69.5 & 69.4 & 69.0 & 68.7 & 68.2 & 67.8 & 67.5 & 67.0 & 66.7 & 66.5 \\
\hline \multicolumn{22}{|c|}{ Mean age by gender } \\
\hline Male $\quad \mathrm{y}$ & yr. & 78.7 & 78.7 & 78.7 & 78.9 & 78.9 & 79.0 & 79.1 & 79.2 & 79.3 & 79.5 & 79.6 & 79.6 & 79.6 & 79.5 & 79.5 & 79.5 & 79.8 & 79.8 & 79.9 & 79.9 \\
\hline Female $\quad \mathrm{y}$ & yr. & 83.6 & 83.6 & 83.6 & 83.7 & 83.7 & 83.7 & 83.9 & 83.9 & 83.9 & 84.0 & 84.0 & 84.0 & 84.0 & 83.9 & 83.9 & 83.8 & 83.7 & 83.7 & 83.6 & 83.6 \\
\hline \multicolumn{22}{|c|}{ Linguistic regions } \\
\hline German & $\%$ & 68.7 & 68.4 & 68.2 & 68.2 & 68.0 & 67.2 & 66.8 & 66.7 & 66.8 & 66.7 & 66.7 & 66.8 & 66.7 & 65.9 & 65.0 & 64.8 & 64.6 & 64.2 & 64.3 & 64.5 \\
\hline French & $\%$ & 25.3 & 25.4 & 25.2 & 24.8 & 24.5 & 25.0 & 25.4 & 25.3 & 25.0 & 25.0 & 24.9 & 24.6 & 24.7 & 25.4 & 26.1 & 26.2 & 27.2 & 27.5 & 27.5 & 27.2 \\
\hline Italian $\quad ?$ & $\%$ & 6.0 & 6.2 & 6.6 & 7.0 & 7.5 & 7.8 & 7.8 & 8.0 & 8.2 & 8.3 & 8.4 & 8.6 & 8.6 & 8.7 & 8.9 & 9.0 & 8.2 & 8.3 & 8.4 & 8.3 \\
\hline \multicolumn{22}{|c|}{ Distribution by frailty levels } \\
\hline Mild $\quad$ ? & $\%$ & 5.4 & 5.6 & 5.7 & 5.6 & 5.7 & 5.7 & 5.7 & 5.7 & 5.8 & 5.8 & 5.9 & 6.0 & 6.4 & 6.8 & 7.1 & 7.3 & 19.0 & 21.6 & 23.2 & 24.1 \\
\hline Moderate & $\%$ & 31.1 & 32.4 & 34.0 & 35.1 & 36.5 & 37.1 & 38.0 & 38.9 & 39.8 & 40.4 & 41.4 & 41.9 & 42.6 & 43.2 & 44.2 & 45.8 & 41.2 & 40.9 & 41.1 & 41.0 \\
\hline Severe & $\%$ & 63.5 & 62.0 & 60.3 & 59.3 & 57.8 & 57.2 & 56.3 & 55.4 & 54.4 & 53.8 & 52.7 & 52.1 & 51.0 & 50.0 & 48.5 & 46.9 & 39.8 & 37.5 & 35.7 & 34.9 \\
\hline \multicolumn{22}{|c|}{ Distribution by types of care } \\
\hline At home & $\%$ & - & - & - & - & - & - & 0.0 & 0.0 & 0.0 & 0.4 & 0.9 & 1.8 & 3.1 & 4.1 & 5.2 & 6.0 & 18.4 & 21.6 & 23.8 & 25.0 \\
\hline In an institution? & & 100.0 & 100.0 & 100.0 & 100.0 & 100.0 & 100.0 & 100.0 & 100.0 & 100.0 & 99.6 & 99.1 & 98.2 & 96.9 & 95.9 & 94.8 & 94.0 & 81.6 & 78.4 & 76.2 & 75.0 \\
\hline Total ? & $\%$ & 100.0 & 100.0 & 100.0 & 100.0 & 100.0 & 100.0 & 100.0 & 100.0 & 100.0 & 99.6 & 99.1 & 98.2 & 96.9 & 95.9 & 94.8 & 94.0 & 81.6 & 78.4 & 76.2 & 75.0 \\
\hline \multicolumn{22}{|c|}{ Total population } \\
\hline $65+\quad t$ & th. 1 & 1044.3 & 1055.1 & 1066.9 & 1079.8 & 1094.3 & 1109.2 & 1131.1 & 1142.5 & 1156.7 & 1174.3 & 1192.5 & 1216.7 & 1245.2 & 1276.4 & 1308.7 & 1334.3 & 1365.2 & 1398.6 & 1432.7 & 1465.6 \\
\hline \multicolumn{22}{|c|}{ Distribution by age groups } \\
\hline 65-79 & $\%$ & 73.2 & 73.4 & 73.7 & 73.9 & 74.1 & 73.7 & 72.9 & 72.6 & 72.4 & 72.1 & 71.8 & 71.6 & 71.6 & 71.6 & 71.6 & 71.8 & 72.0 & 72.1 & 72.2 & 72.1 \\
\hline $80+$ & $\%$ & 26.8 & 26.6 & 26.3 & 26.1 & 25.9 & 26.3 & 27.1 & 27.4 & 27.6 & 27.9 & 28.2 & 28.4 & 28.4 & 28.4 & 28.4 & 28.2 & 28.0 & 27.9 & 27.8 & 27.9 \\
\hline \multicolumn{22}{|c|}{ Prevalence rates: number of registered old-age care beneficiaries divided by the total population } \\
\hline & $\%$ & 3.2 & 3.3 & 3.3 & 3.4 & 3.4 & 3.5 & 3.6 & 3.6 & 3.6 & 3.6 & 3.6 & 3.6 & 3.5 & 3.4 & 3.4 & 3.4 & 4.0 & 4.0 & 4.0 & 4.0 \\
\hline \multicolumn{22}{|c|}{ Distribution by age groups } \\
\hline 65-79 & $\%$ & 1.5 & 1.6 & 1.6 & 1.7 & 1.7 & 1.7 & 1.7 & 1.7 & 1.7 & 1.7 & 1.7 & 1.7 & 1.7 & 1.7 & 1.7 & 1.7 & 1.9 & 1.9 & 1.9 & 2.0 \\
\hline $80+$ & $\%$ & 7.8 & 7.9 & 8.0 & 8.3 & 8.3 & 8.5 & 8.5 & 8.4 & 8.4 & 8.5 & 8.4 & 8.3 & 8.1 & 7.9 & 7.8 & 7.9 & 9.3 & 9.4 & 9.4 & 9.3 \\
\hline
\end{tabular}

Table 1: Descriptive statistics on old-age care benefits and prevalence rates for the years from 1995 to 2014. 


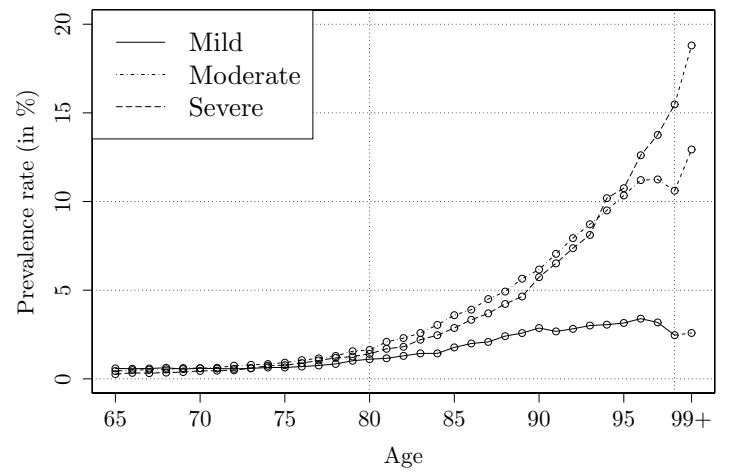

(a) Frailty levels

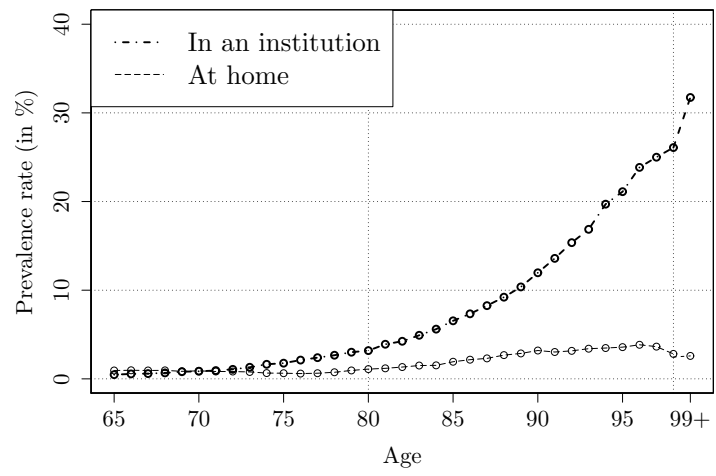

(b) Types of care

Figure 2: Prevalence rates by age, by frailty levels and by types of care in 2014 .

figure, we remark heterogeneity among cantons in the prevalence rates around the national average of $3.9 \%$. The canton TI (see Footnote 1 for the abbreviations) is the canton with the highest prevalence rate $(6.5 \%)$ while the canton TG shows the lowest prevalence rate $(2.8 \%)$. We also observe that most of the German-speaking cantons (e.g., ZH, BE) rank below the average prevalence rate and most of the French-speaking cantons (e.g., VD, GE) are above this value. The only Italian-speaking canton (TI) leads the ranking. These differences may be explained by regional and cultural differences but may also result from a different registration behavior or information about the benefits in the respective regions (Weaver, 2012).

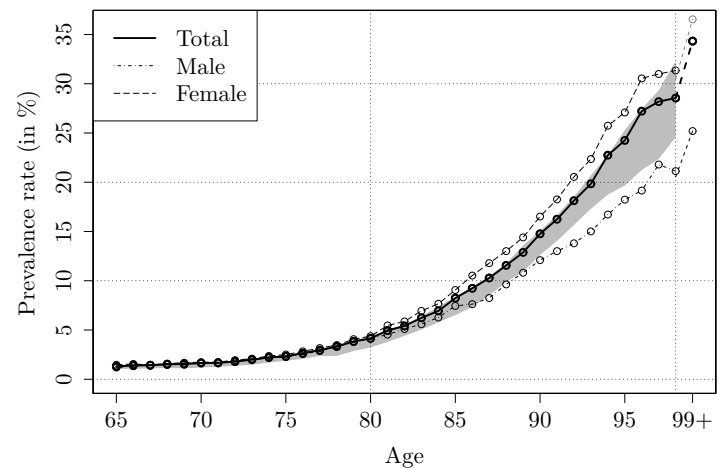

(a) Gender

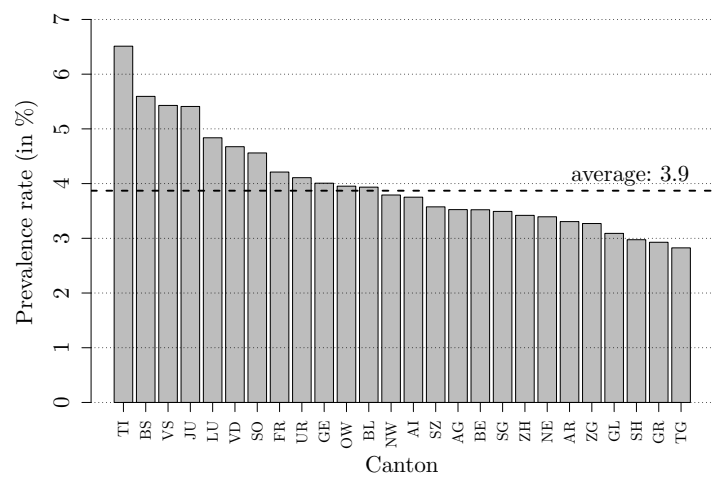

(b) Cantons

Figure 3: Prevalence rates by age, by gender and by cantons in 2014 .

\subsection{Historical and predicted demographic development}

In this last part of Section 2, we discuss the historical and the future headcount of the population aged 65 years or older in Switzerland. The historical values correspond to the years before 2014 for which the statistics are available from the FSO (cf. Footnote 3). For the years between 2015 and 2045, we consider three scenarios produced by the FSO, namely A-00-2015, B-00-2015 and C-00-2015 (see FSO, 2015). The scenario A-00-2015 represents the reference scenario. Male life expectancy is assumed to be 81.2, 84.2 and 86.2 years in 2015, 2030 and 2045, respectively, 
while female life expectancy takes the values of 85.1, 87.6 and 89.4 years. Further, the population aged over 65 years is about 1.5, 2.2 and 2.7 million in 2015, 2030 and 2045. The population aged over 80 years reaches $0.4,0.7$ and 1.1 million. The scenarios B-00-2015 and C-00-2015 provide a high respectively low population projection. Thereby the assumptions on the life expectancy, the immigration and the emigration are changed. Table 2 provides a summary of the parameter values. In the high scenario, the population aged $65+$ reaches 1.5, 2.2 and 2.8 million for the years 2015, 2030 and 2045 while the population aged over 80 years counts 0.4 , 0.7 and 1.1 million elderly. Finally, in the low scenario the $65+$ population projection is 1.5 , 2.1 and 2.6 million and $0.4,0.7$ and 1.0 persons are projected for ages over 80 years.

\begin{tabular}{|c|c|c|c|c|c|c|c|c|c|}
\hline & \multicolumn{3}{|c|}{ Reference } & \multicolumn{3}{|c|}{ Low } & \multicolumn{3}{|c|}{ High } \\
\hline & 2015 & 2030 & 2045 & 2015 & 2030 & 2045 & 2015 & 2030 & 2045 \\
\hline Male life expectancy (in yrs.) & 81.2 & 84.2 & 86.2 & 81.0 & 83.6 & 85.3 & 81.3 & 84.5 & 86.9 \\
\hline Female life expectancy (in yrs.) & 85.1 & 87.6 & 89.4 & 85.0 & 87.1 & 88.6 & 85.3 & 87.9 & 90.1 \\
\hline Immigration (in th.) & 175 & 155 & 125 & 160 & 130 & 110 & 190 & 180 & 140 \\
\hline Emigration (in th.) & 95 & 95 & 95 & 90 & 90 & 90 & 100 & 100 & 100 \\
\hline Total population $65+$ (in th.) & 1501 & 2174 & 2690 & 1501 & 2138 & 2563 & 1503 & 2208 & 2810 \\
\hline Total population $80+$ (in th.) & 420 & 686 & 1055 & 420 & 671 & 998 & 421 & 701 & 1108 \\
\hline
\end{tabular}

Table 2: Main characteristics of the FSO (2015) scenarios A-00-2015 (reference), B-002015 (high), C-00-2015 (low).

In Figure 4, we illustrate the evolution of the population pyramid in Switzerland by age and by gender from 1985 to 2045 . While the figures for 1985, 1995 and 2005 stem from the historical statistics, the predictions for the years from 2015 to 2045 are taken from the reference scenario (A-00-2015). We observe a particular shape in the curves describing the population by ages for the different years. For the year 1985, the number of persons aged 66 years (the same holds for the ages of 67 through 70 years) is lower than the number of elderly aged 71 years. We also observe the presence of such irregularity in the years 1995 and 2005 for the population aged between 75 and 80 years, and the population aged from 85 to 90 years, respectively. The explanation for the presence of these drops in the population comes both form the Second World War and hard life conditions (FSO, 1998). On the other hand, the year 2015 is characterized by the presence of the retiring baby-boomer generation at the ages from 65 to 75 where the population pyramid shows a hump. This specificity also appears at higher ages for the years 2025 and 2035 . In 2045, we find an important hump in the population aged from 75 to 85 years. Given that LTC needs affect to a large extent elderly aged $80+$ (cf. Section 2.3) the demographic development adds to the challenging conditions.

We report the size of the population aged $65+$ and $80+$ and compare the evolution between the years 1985 and 2015, the years 2015 and 2030 and the years 2015 and 2045 in Table 3 . The 1985 census counts 367 thousand males and 552 thousand females aged $65+$ among which 67 thousand males and 143 thousand females are aged 80+. In 2015, we count a total of 1501 thousand persons aged over 65 years and 1055 thousand persons aged over 80 years. The forecasts for the year 2045 predict a population composed by 2690 thousand $65+$-individuals and 1055 thousand $80+$-individuals. These numbers claim for a faster increase of the sub-population aged $80+$ than the overall 65+-population. In the 30-year periods 1985-2015 and 2015-2045, the population aged $65+$ is expected to increase by about $63 \%$ and $79 \%$, respectively. The population aged $80+$ presents significantly higher increases with multiplicative factors of 2.00 and 2.51 . 


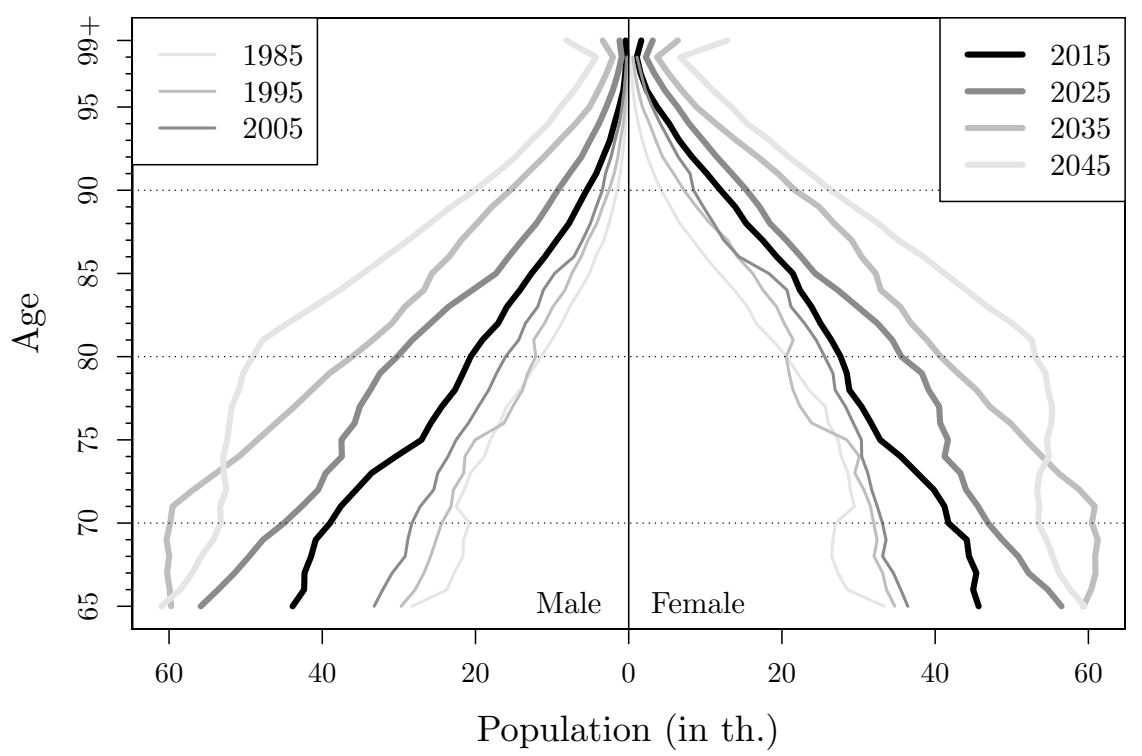

Figure 4: Evolution of the population pyramid in Switzerland by age and by gender from 1985 to 2045 (projections illustrated with thick lines stem from the reference scenario).

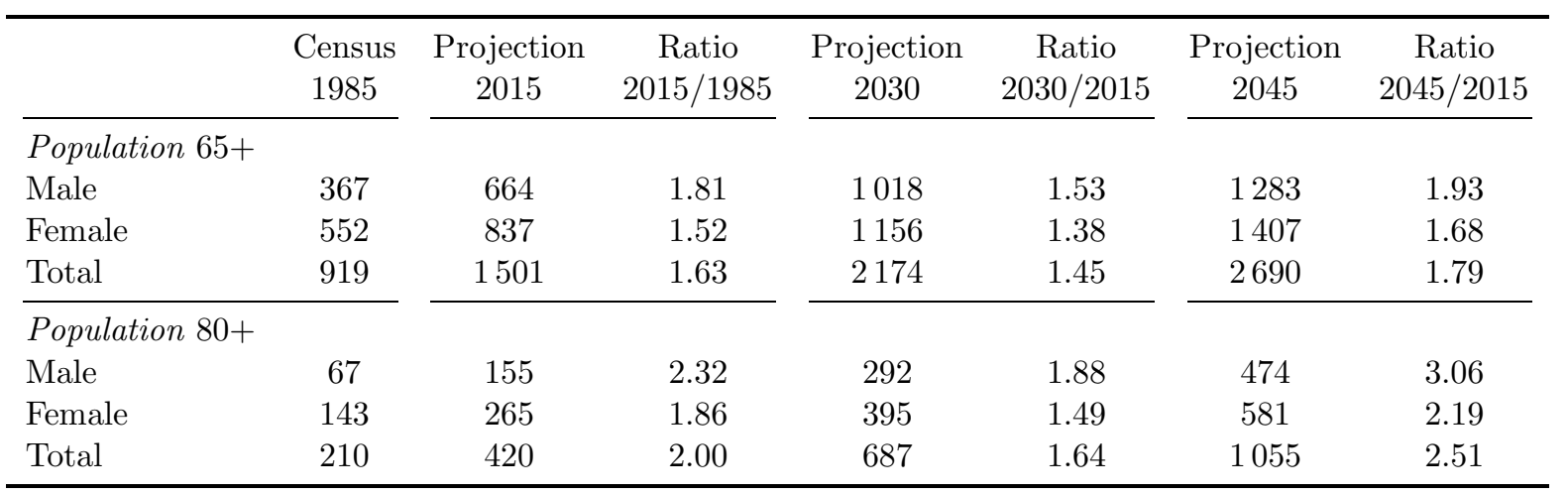

Table 3: Size of the population aged $65+$ and $80+$ (in th.) and illustration of the ratios between the years 2015/1985, 2030/2015 and 2045/2015.

\section{Model framework}

Our objective is to determine the effect of selected drivers on prevalence rates log-linear regression models. We first consider the total prevalence and then distinguish the rates by frailty levels and types of care. In the following, we define the prevalence rates, the available characteristics related to them (Section 3.1) and propose the regression models (Section 3.2).

\subsection{Definition of the prevalence rates and study of the characteristics}

\section{Prevalence rates}

In our study, we distinguish six prevalence rates. First, we analyze the total prevalence rates $\pi$ which are the number of old-age care beneficiaries divided by the total population as presented in Table 1 and Figures $1 \mathrm{~b}$ and 3. Building on the observations from Figure 2, we separately consider the specific prevalence rates related to the various frailty levels and types of care. 
More precisely, we describe dependency using five further variables. The prevalence rates for the mild (1), the moderate (2) and the severe (3) dependency are denoted by $\pi^{1}, \pi^{2}$ and $\pi^{3}$, respectively. For the types of care, we use the notations $\pi^{\mathrm{a}}$ and $\pi^{\mathrm{b}}$ to differentiate prevalence rates between elderly cared for at home (a) to those cared for in an institution (b). With this approach, we provide more detailed results along acuity levels and types of care (Czado and Rudolph, 2002). While all acuity levels are highly relevant for the concerned individual, the information on the type of care is particularly important for organizing care facilities and for financing them. Our decision to consider separate models is further motivated by the observed collinearity between the frailty levels and the types of care, i.e., severely dependent persons will be mainly cared for in an institution while midly dependent persons mostly stay at home. The prevalence rates summarized in Table 4 will serve as dependent variables in our regression models.

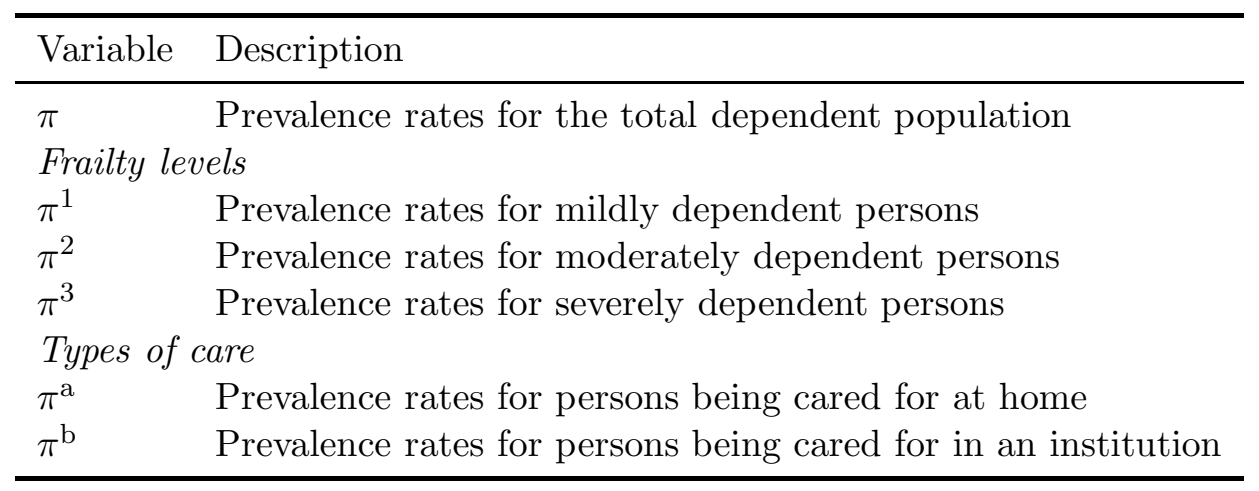

Table 4: Description of the dependent variables.

We now address the expected effect of selected variables on the prevalence rates. In line with the descriptive statistics laid out in Section 2, we introduce two variables directly related to the individuals' age and gender. These will be the first independent variables in our models (see the summary in Table 5).

\section{Age}

Many studies have evidenced a positive dependence between the age and the prevalence rates (Yip et al., 2006; Meguro et al., 2012; Biessy, 2016). At higher ages, the increase in prevalence rates finds different explanations. On a first hand, the natural aging process explains the appearance of difficulties in accomplishing daily life activities (Xue, 2011). In the study of Han et al. (2013), the effect of the age on functional disability remains even when accounting for other risk factors like depression, cognitive impairment and physical frailty. On a second hand, the development of cognitive and physical diseases leads to consequent limitations in ADLs and requires LTC (see, e.g., Monod-Zorzi et al., 2007). For example, the study of Callahan et al. (2012) identifies persons affected by dementia as the main consumers of family caregivers. Following those previous studies and the own observations from Figure 3a (see Section 2.3), we expect a significant positive relationship between the age and the prevalence rates. Since at higher ages, the underlying population becomes very small at the canton level (see below), we will group the ages into age classes by steps of five years in order to get representative values for the prevalence rates. 


\section{Gender}

Besides the natural aging process, previous research has identified a significant gender effect. For example, De Meijer et al. (2011) and Fong et al. (2017) find that women require more LTC than male since they spend more time in dependency. In Crimmins et al. (2011), based on three international surveys (SHARE, ELSA and HRS), the authors observe more limitations in ADLs for women than for men. They also report an increase of the gender difference at higher ages (we briefly discuss the age-gender interaction in Sections 3.2 and 4.2). Thus, we introduce the gender as the second independent variable of interest. Building on these findings above and the statistics presented in Section 2, we expect our models to exhibit significant differences in prevalence rates by gender with women showing higher rates.

Beyond age and gender, we consider the inclusion of the canton of residence, the linguistic region, the calendar year of the observation and three sub-periods within the 1995-2014 time frame.

\section{Canton and linguistic region}

By examining the 26 cantons, we have observed differences in the prevalence rates (see Figure $3 \mathrm{~b}$ ). In his work, Cosandey (2016) discusses institutional care services and reveals differences between cantons. This is also observed in El Bernoussi and Rockinger (2017) when considering the evolution of LTC demand. Given the differing demographic structure among the cantons (Cosandey, 2016), we also expect differences in the population forecasts (see also Section 5). However, the results will be complex to interpret since many hidden factors can affect the outcome (e.g., cultural aspects linked to care provided by relatives, cantonal decisions on the allocation and the amounts of LTC allowance, heterogeneity in the medical assessment). Three linguistic regions represent the clusters of cantons along the three main national languages German, French and Italian (see Footnote 1). This categorization is commonly used in Switzerland and can also as a proxy of cultural differences. For example, Eugster et al. (2011) find significant differences in social insurance demand depending on the linguistic region. This is reinforced by Gentili et al. (2017) who find that people residing in the French-speaking region show higher usage of at-home care in comparison to elderly living in the German-speaking area. We expect that both Latin-speaking regions (French and Italian) will report higher prevalence rates than the German-speaking region since we observe the major French-speaking cantons (FR, GE, VS, VD) and the Italian-speaking canton (TI) exceeding the national average (see Figure 3b).

\section{Year and period}

Finally, we consider the inclusion of the year variable identifying the calendar year of the observation and the period variable representing a cluster of years. These temporal variables allow for pointing out changes in the LTC allowance system. In fact, we observe critical changes in the years 2001 and 2011 (Weaver, 2012). The year 2001 represents the recognition for care provided at home while the year 2011 identifies the implementation of a new LTC financing scheme which also recognizes mildly dependent persons cared for at home (see also Section 2.2). Based on these observations, we build the sub-period variable to consider the three time frames 19952001, 2002-2010 and 2011-2014. The boundary years 2001 and 2011 have the characteristics laid out above while the years 1995 and 2014 stem from the limits of the available data. The descriptive statistics presented in Figure 1 let us expect a significant time effect on the prevalence 
rates over the years from 1995 to 2010 and a non-significant effect over the years from 2011 to 2014.

\begin{tabular}{ll}
\hline Variable & Description \\
\hline$A G E$ & Age class of the observation: $65-69,70-74, \ldots, 90-94,95+$ \\
GENDER & Gender of the observation: male, female \\
YEAR & Calendar year of the observation: 1995, ., 2014 \\
PERIOD & Time period of the observation: 1995-2001, 2002-2010, 2011-2014 \\
CANTON & Canton in which is located the observation (see Footnote 1) \\
LING & Linguistic region in which is located the observation: DE, FR, IT \\
\hline
\end{tabular}

Table 5: Description of the independent variables.

\subsection{Regression models}

We explain the relationship between the prevalence rates and the independent variables described above through a log-linear ordinary least square (OLS) regression model. This type of model is particularly suited for representing exponential relations between dependent and independent variables (Stock and Watson, 2012). In our case, the discussion in Section 2.3 has evidenced an exponential link between the prevalence rates and the dependent persons' age. We study this relation through the regression equations defined below. The log-linear regression framework rarely applies for dependent variables expressed as a ratio (Wooldridge, 2013). In the sequel, we use such regression for explaining the logarithm of the prevalence rates which are, by definition, ratio data. We show our results in Sections 4.2 and 4.3 to be highly significant and the models yield high values of adjusted $R$-squared (cf. Tables 6 and 7). This reveals ex-post that the log-linear regression performs well (see the discussion in Section 4.4).

Within the explanatory variables, the age is included through the classes $j \in\{1, \ldots, 6\}$ coded in the variable $A G E_{i}^{j}(j=1$ for ages $70-74, j=2$ for ages $75-79$, and so on until $j=6$ for ages $95+$ ). Further, we consider the gender GENDER $R_{i}$, the observation period PERIOD $D_{i}^{k}$, the linguistic region $L I N G_{i}^{l}$ and the canton of residence $C A N T O N_{i}^{m}$ effects on the prevalence rates. The variable $P E R I O D_{i}^{k}$ aggregates the variable $Y E A R_{i}$ in three sub-periods (cf. Table 5 ). We use the subscript $i$ for referring to the observations while the index $k \in\{1,2\}$ identifies the periods 1995-2001 $(k=1)$ and 2002-2010 $(k=2)$. In $L I N G_{i}^{l}$, the index $l \in\{1,2\}$ relates to the French- $(l=1)$ and Italian- $(l=2)$ speaking regions. Finally, the index $m \in\{1,2, \ldots, 25\}$ in $C A N T O N_{i}^{m}$ defines the canton of residence (see Footnote 1). For the purpose of our analysis, the above categorical variables have to be interpreted with respect to a baseline. Observations from the age class 65-69 are the baseline for $A G E_{i}^{j}$, men are the baseline for GENDER the period 2011-2014 is the baseline for $P E R I O D_{i}^{k}$, the German linguistic region (DE) is the baseline for $L I N G_{i}^{l}$ and the canton $\mathrm{ZH}$ is the baseline for $C A N T O N_{i}^{m}$. We denote by $\epsilon_{i}$ the error term in the regressions.

\section{Regression on the period from 1995 to 2014}

The below Equation (1) expresses the logarithm of the total prevalence rate $\left(\log \pi_{i}\right)$ as a linear combination of the intercept $\left(\beta_{0}\right)$, the observation's age class $\left(A G E_{i}^{j}\right)$, its gender $\left(G E N D E R_{i}\right)$, the observation period $\left(P E R I O D_{i}^{k}\right)$ and the linguistic region $\left(L I N G_{i}^{l}\right)$ : 


$$
\log \pi_{i}=\beta_{0}+\sum_{j} \beta_{1}^{j} A G E_{i}^{j}+\beta_{2} G E N D E R_{i}+\sum_{k} \beta_{4}^{k} P E R I O D_{i}^{k}+\sum_{l} \beta_{5}^{l} L I N G_{i}^{l}+\epsilon_{i} .
$$

Due to their lower mortality, particularly at higher ages, female live more years than males in dependence (Mathers, 1996; Mathers et al., 2001; Monod-Zorzi et al., 2007; Fong et al., 2017). Further, Monod-Zorzi et al. (2007) find that diseases leading to dependence appear at different ages for both genders. Therefore, we measure the interaction effect between the age class and the gender by adding the term $\sum_{n} \beta_{7}^{n} A G E_{i}^{n} \times G E N D E R_{i}$ in Equation (1) leading to the model:

$$
\begin{aligned}
\log \pi_{i}= & \beta_{0}+\sum_{j} \beta_{1}^{j} A G E_{i}^{j}+\beta_{2} G E N D E R_{i}+\sum_{k} \beta_{4}^{k} P E R I O D_{i}^{k}+\sum_{l} \beta_{5}^{l} L I N G_{i}^{l} \\
& +\sum_{n} \beta_{7}^{n} A G E_{i}^{n} \times G E N D E R_{i}+\epsilon_{i} .
\end{aligned}
$$

In a next step, we aim to refine the regression model (1) by considering the canton of residence instead of the linguistic region, leading to Equation (3):

$$
\log \pi_{i}=\beta_{0}+\sum_{j} \beta_{1}^{j} A G E_{i}^{j}+\beta_{2} G E N D E R_{i}+\sum_{k} \beta_{4}^{k} P E R I O D_{i}^{k}+\sum_{m} \beta_{6}^{m} \operatorname{CANTON}_{i}^{m}+\epsilon_{i} .
$$

Thereafter, we stratify the dataset in the three sub-periods defined above and run the regression model (4) on each of them using the corresponding subset of data. We consider the effect of the age, the gender, the calendar year of the observation and the canton of residence on the logarithm of the prevalence rate:

$$
\log \pi_{i}=\beta_{0}+\sum_{j} \beta_{1}^{j} A G E_{i}^{j}+\beta_{2} G E N D E R_{i}+\beta_{3} Y E A R_{i}+\sum_{m} \beta_{6}^{m} C A N T O N_{i}^{m}+\epsilon_{i} .
$$

By considering the three sub-periods separately, we first set aside heterogeneity due to the changes in LTC allowances (see Sections 2.2 and 3.1) and then quantify the year effect within each period.

\section{Regression on the frailty level and the type of care prevalence}

Basing our further analyses on the most recent period 2011-2014, we distinguish more specific models. A first set of models takes into account the frailty levels by considering the severity of the registered dependence. Recall that the three dependency states are distinguished by their respective severity mild (1), moderate (2) or severe (3). The second set focuses on the types of care received. In these models, we identify the two groups of persons cared for at home (a) and in an institution (b). The regression models (5.s) formalize the expression making use of the specific prevalence rates $\pi_{i}^{(s)}$ as dependent variables (Table 4$)$. We consider:

$$
\log \pi_{i}^{(s)}=\beta_{0}+\sum_{j} \beta_{1}^{j} A G E_{i}^{j}+\beta_{2} G E N D E R_{i}+\sum_{m} \beta_{6}^{m} C A N T O N_{i}^{m}+\epsilon_{i}
$$

where $s \in\{1,2,3\}$ or $\{\mathrm{a}, \mathrm{b}\}$. In the label (5.s), the index $s$ takes the values 1,2 or 3 for the mild, moderate or severe states. It takes the values a or b for care received at home or in an institution. 


\section{Application of the models and empirical results}

\subsection{Available data and implementation}

The dataset for our study consists of the $65+$ elderly registered for receiving the OASI allowance due to limitations in ADLs collected by the CCO and of the Swiss population census produced by the FSO (see Section 2). The CCO longitudinal dataset covers the $65+$ population requesting the OASI allowance due to LTC needs in the period from 1995 to 2014. This dataset contains the total registered population being cared for in an institution. Meanwhile, it only offers a limited picture of persons receiving care at home. This limitation essentially follows from changes in law on the recognition of at home care (see Section 2.2) and subsequently from the request process. In fact, each individual has to send a request to the OASI in order to receive the allowance. While this process is mostly automatic for persons receiving LTC in an institution, many persons receiving care at home are not aware of such allowance and may not request it (Weaver, 2012). For each calendar year, we evaluate the total number of registered dependent at the end of the year including information on their age, gender, canton and acuity level (frailty level and type of care).

The observations in our data are the prevalence rates for each calendar year by age, canton, frailty level and type of care. They are obtained from the ratio of the number of registered dependent elderly over the total population from the FSO population census. ${ }^{6}$ Overall descriptive statistics are presented at the bottom of Table 1 . The theoretical number of observations is given by the combination of all the values taken by the independent variables. We account for 7280 bins for the total prevalence rate $\pi$, obtained by combinations of the seven age classes (see Table 5), the two genders (male and female), the 26 cantons (see Footnote 1) and the 20 calendar years (1995 to 2014). The total number of bins comes from the multiplication $7 \times 2 \times 26 \times 20=7280$. Since prevalence rates are not available for all ages in all cantons and in all calendar years, we are left with $N=7244$ observations for our study. We are thus missing less than $0.5 \%$ of observations when relating them to the total number of bins. For the specific prevalence rates $\pi^{(s)}, s \in\{1,2,3\}$ or $s \in\{\mathrm{a}, \mathrm{b}\}$, we limit our analysis to the sub-period from 2011 to 2014. Thus, the theoretical number of bins decreases to 1456 . The difference between this number and the number of empirical observations $N$ (see Tables 6 and 7 ) informs on missing values. Each observation enters the regression models defined in Section 3.2 and is referred to with the index $i$.

\subsection{Results of the regression on the period from 1995 to 2014}

Table 6 reports the estimates for the coefficients of the regression models (1) to (4). As mentioned in Section 3, we apply Equations (1) and (3) on the period 1995-2014 while the Equations (4) and $\left(4^{\prime}\right)$ are based on the three sub-periods (1995-2001, 2002-2010 and 2011-2014). Equation $\left(4^{\prime}\right)$ will be introduced at the end of this section after considering the results from the model (4). We report the independent variables in the first column. For categorical and

\footnotetext{
${ }^{6}$ The historical census on the population for the years 1995-2014 is built upon the aggregation of two publicly available datasets, the first one covering the years of interest from 1995 to 2010 and the second one covering the years from 2010 to 2014. For the year 2010 which appears in both datasets, we take the average values between the datasets. Furthermore, the first dataset covers the ages from 65 to 98 years separately and comprises a $99+-$ category while the second dataset covers the ages from 65 to 99 and has a 100+-category. We merge the datasets and build a 99+-category corresponding to the sum of the 99+ in the first dataset and the classes 99 and $100+$ from the second dataset.
} 


\begin{tabular}{|c|c|c|c|c|c|c|c|c|c|c|c|}
\hline \multirow{2}{*}{$\begin{array}{l}\text { Model } \\
\text { Period } \\
\text { Intercept }\end{array}$} & $\begin{array}{c}(1) \\
1995-2014 \\
\end{array}$ & $\begin{array}{c}(2) \\
1995-2014\end{array}$ & $\begin{array}{c}(3) \\
1995-2014\end{array}$ & \multicolumn{2}{|c|}{$\begin{array}{c}(4) \\
1995-2001 \\
\end{array}$} & \multicolumn{2}{|c|}{$\begin{array}{c}(4) \\
2002-2010\end{array}$} & \multicolumn{2}{|c|}{$\begin{array}{c}(4) \\
2011-2014 \\
\end{array}$} & \multicolumn{2}{|c|}{$\begin{array}{c}\left(4^{\prime}\right) \\
2011-2014 \\
\end{array}$} \\
\hline & $-4.496 * * *(.012)$ & $-4.354 * * *(.014)$ & $-4.622 * * *(.019)$ & $-31.428 * * *$ & $(5.724)$ & $15.260 * * *$ & $(3.341)$ & -8.516 & $(9.349)$ & $-4.510 * * *$ & * $(.030)$ \\
\hline \multicolumn{12}{|c|}{ Age (baseline: 65-69) } \\
\hline $70-74$ & $0.265 * * *(.012)$ & $0.270 * * *(.017)$ & $0.267 * * *(.012)$ & $0.303 * * *$ & $(.021)$ & $0.272 * * *$ & $(.016)$ & $0.195 * * *$ & (.019) & $0.195 * * *$ & * (.019) \\
\hline $75-79$ & $0.763 * * *(.013)$ & $0.177^{* * *}(.017)$ & $0.763 * * *(.012)$ & $0.819^{* * *}$ & $(.021)$ & $0.745 * * *$ & $(.016)$ & $0.702 * * *$ & (.019) & $0.702 * * *$ & * (.019) \\
\hline $80-84$ & $1.374 * * *(.013)$ & $1.223 * * *(.017)$ & $1.374 * * *(.012)$ & $1.423 * * *$ & $(.021)$ & $1.354 * * *$ & $(.016)$ & $1.333^{* * *}$ & $(.019)$ & $1.333^{* * *}$ & $*(.019)$ \\
\hline $85-89$ & $1.967 * * *(.013)$ & $1.737 * * *(.017)$ & $1.967 * * *(.012)$ & $2.012 * * *$ & $(.021)$ & $1.959 * * *$ & $(.016)$ & $1.906 * * *$ & $(.019)$ & $1.906 * * *$ & $*(.019)$ \\
\hline $90-94$ & $2.503 * * *(.013)$ & $2.233 * * *(.017)$ & $2.503 * * *(.012)$ & $2.559 * * *$ & $(.021)$ & $2.492 * * *$ & $(.016)$ & $2.428 * * *$ & $(.019)$ & $2.428 * * *$ & $*(.019)$ \\
\hline $95+$ & $2.875 * * *(.013)$ & $2.567 * * *(.017)$ & $2.874^{* * *}(.012)$ & $2.959 * * *$ & $(.022)$ & $2.808 * * *$ & $(.016)$ & $2.869 * * *$ & $(.020)$ & $2.869 * * *$ & * $(.020)$ \\
\hline \multicolumn{12}{|c|}{ Gender (baseline: Male) } \\
\hline Female & $0.248^{* * *}(.007)$ & $-0.036 *$ & $0.248 * * *(.006)$ & $0.279 * * *$ & $(.011)$ & $0.246 * * *$ & $(.009)$ & $0.200 * * *$ & $(.010)$ & $0.200 * * *$ & * $(.010)$ \\
\hline \multicolumn{12}{|c|}{ Period (baseline: 2011-2014) } \\
\hline $1995-2001$ & $-0.151 * * *(.009)$ & $-0.152 * * *(.009)$ & $-0.152 * * *(.009)$ & & & & & & & & \\
\hline $2002-2010$ & $-0.140 * * *(.010)$ & $-0.140 * * *(.009)$ & $-0.140 * * *(.008)$ & & & & & & & & \\
\hline Year & & & & $0.013 * * *$ & $(.003)$ & $-0.010 * * *$ & $(.002)$ & 0.002 & $(.005)$ & & \\
\hline \multicolumn{12}{|c|}{ Linguistic region (baseline: DE) } \\
\hline FR & $0.155^{* * *}(.008)$ & $0.156^{* * *}(.008)$ & & & & & & & & & \\
\hline IT & $0.425 * * *(.018)$ & $0.427 * * *(.017)$ & & & & & & & & & \\
\hline \multicolumn{12}{|c|}{ Canton (baseline: ZH) } \\
\hline $\mathrm{AG}$ & & & $0.114 * * *(.022)$ & $0.098 *$ & $(.041)$ & $0.139 * * *$ & $(.031)$ & $0.084 *$ & $(.037)$ & $0.084 *$ & $(.037)$ \\
\hline AI & & & $0.165^{* * *}(.022)$ & 0.056 & $(.042)$ & $0.281 * * *$ & $(.031)$ & $0.089 *$ & $(.038)$ & $0.089 *$ & $(.038)$ \\
\hline $\mathrm{AR}$ & & & $-0.016 \quad(.022)$ & -0.049 & $(.041)$ & 0.028 & $(.031)$ & -0.060 & $(.037)$ & -0.060 & $(.037)$ \\
\hline $\mathrm{BE}$ & & & $0.152 * * *(.022)$ & $0.270 * * *$ & $(.041)$ & $0.120 * * *$ & $(.031)$ & 0.019 & $(.037)$ & 0.019 & $(.037)$ \\
\hline BL & & & $0.270 * * *(.022)$ & $0.346 * * *$ & $(.041)$ & $0.253 * * *$ & $(.031)$ & $0.174 * * *$ & $(.037)$ & $0.174 * * *$ & $*(.037)$ \\
\hline BS & & & $0.232 * * *(.022)$ & $0.140^{* * *}$ & $(.041)$ & $0.262 * * *$ & $(.031)$ & $0.328 * * *$ & $(.037)$ & $0.328^{* * *}$ & * $(.037)$ \\
\hline FR & & & $0.476 * * *(.022)$ & $0.501 * * *$ & $(.041)$ & $0.522 * * *$ & $(.031)$ & $0.327 * * *$ & $=(.037)$ & $0.327 * * *$ & $*(.037)$ \\
\hline GE & & & $-0.005 \quad(.022)$ & -0.041 & $(.041)$ & -0.028 & $(.031)$ & $0.107 * *$ & $(.037)$ & $0.107 * *$ & $(.037)$ \\
\hline GL & & & $0.101 * * *(.022)$ & $0.190 * * *$ & $(.041)$ & $0.123 * * *$ & $(.031)$ & $-0.106 * *$ & $(.038)$ & $-0.106 * *$ & $(.038)$ \\
\hline GR & & & $0.076^{* * *}(.022)$ & $0.217 * * *$ & $(.041)$ & 0.056 & $(.031)$ & $-0.127 * * *$ & $(.037)$ & $-0.127 * * *$ & $*(.037)$ \\
\hline $\mathrm{JU}$ & & & $0.522 * * *(.022)$ & $0.658 * * *$ & $(.041)$ & $0.468 * * *$ & $(.031)$ & $0.406^{* * *}$ & $=(.037)$ & $0.406 * * *$ & * $(.037)$ \\
\hline $\mathrm{LU}$ & & & $0.400 * * *(.022)$ & $0.409 * * *$ & $(.041)$ & $0.409 * * *$ & $(.031)$ & $0.366 * * *$ & $(.037)$ & $0.366 * * *$ & $*(.037)$ \\
\hline $\mathrm{NE}$ & & & $0.053^{*} \quad(.022)$ & $0.127 * *$ & $(.041)$ & 0.014 & $(.031)$ & 0.010 & $(.037)$ & 0.010 & $(.037)$ \\
\hline NW & & & $0.023 \quad(.022)$ & $0.091 *$ & $(.041)$ & $-0.079 *$ & $(.031)$ & $0.133^{* * *}$ & $(.037)$ & $0.133 * * *$ & $*(.037)$ \\
\hline OW & & & $0.126 * * *(.022)$ & 0.080 & $(.041)$ & $0.152 * * *$ & $(.031)$ & $0.148^{* * *}$ & $(.037)$ & $0.148 * * *$ & * $(.037)$ \\
\hline $\mathrm{SG}$ & & & $0.085 * * *(.022)$ & $0.118 * *$ & $(.041)$ & $0.083 * * *$ & $(.031)$ & 0.034 & $(.037)$ & 0.034 & $(.037)$ \\
\hline $\mathrm{SH}$ & & & $0.018 \quad(.022)$ & $0.115^{* *}$ & $(.041)$ & 0.011 & $(.031)$ & $-0.136 * * *$ & $=(.037)$ & $-0.136 * * *$ & $*(.037)$ \\
\hline $\mathrm{SO}$ & & & $0.233^{* * *}(.022)$ & $0.153^{* * *}$ & $(.041)$ & $0.276 * * *$ & $(.031)$ & $0.275 * * *$ & $=(.037)$ & $0.275 * * *$ & $*(.037)$ \\
\hline $\mathrm{SZ}$ & & & $0.174 * * *(.022)$ & $0.226^{* * *}$ & $(.041)$ & $0.157 * * *$ & $(.031)$ & $0.123 * *$ & $(.037)$ & $0.123 * *$ & $(.037)$ \\
\hline TG & & & $-0.027 \quad(.022)$ & 0.021 & $(.041)$ & -0.009 & $(.031)$ & $-0.152 * * *$ & $(.037)$ & $-0.152 * * *$ & $*(.037)$ \\
\hline TI & & & $0.552 * * *(.022)$ & $0.478 * * *$ & $(.041)$ & $0.605^{* * *}$ & $(.031)$ & $0.562 * * *$ & $(.037)$ & $0.562 * * *$ & $*(.037)$ \\
\hline UR & & & $0.311 * * *(.022)$ & $0.342 * * *$ & $(.041)$ & $0.367 * * *$ & $(.031)$ & $0.127 * * *$ & $(.037)$ & $0.127 * * *$ & $*(.037)$ \\
\hline VD & & & $0.218^{* * *}(.022)$ & $0.345^{* * *}$ & $(.041)$ & $0.209 * * *$ & $(.031)$ & $0.279 * * *$ & $(.037)$ & $0.279 * * *$ & $*(.037)$ \\
\hline VS & & & $0.425 * * *(.022)$ & $0.445^{* * *}$ & $(.041)$ & $0.386 * * *$ & $(.031)$ & $0.475 * * *$ & $(.037)$ & $0.475 * * *$ & $*(.037)$ \\
\hline ZG & & & $-0.043 . \quad(.022)$ & -0.077 & $(.041)$ & -0.050 & $(.031)$ & 0.030 & $(.037)$ & 0.030 & $(.037)$ \\
\hline \multicolumn{12}{|c|}{ Age-Gender (baseline: $65-69 \times$ Male) } \\
\hline $70-74 \times$ Female & & $-0.01 \quad(.024)$ & & & & & & & & & \\
\hline $75-79 \times$ Female & & $0.102 * * *(.024)$ & & & & & & & & & \\
\hline $80-84 \times$ Female & & $0.300 * * *(.024)$ & & & & & & & & & \\
\hline $85-89 \times$ Female & & $0.460 * * *(.024)$ & & & & & & & & & \\
\hline 90-94 × Female & & $0.540 * * *(.024)$ & & & & & & & & & \\
\hline $95+\times$ Female & & $0.604 * * *(0.24)$ & & & & & & & & & \\
\hline$N$ & 7244 & $\overline{7244}$ & 7244 & 2526 & & 3266 & & 1452 & & 1452 & \\
\hline Adj. $R^{2}$ & 0.924 & 0.936 & 0.941 & 0.932 & & 0.947 & & 0.965 & & 0.965 & \\
\hline
\end{tabular}

Note: . $p<0.1, * p<0.05,{ }^{* *} p<0.01,{ }^{* * *} p<0.001$

Numbers in parenthesis represent standard deviations.

Table 6: Results for the regression models (1) to (4) and $\left(4^{\prime}\right)$.

binary variables the baseline is given. The last two rows of the table present the number of observations $(N)$ and the adjusted $R$-squared (Adj. $R^{2}$ ). Next to the estimates, the standard deviation is given in parenthesis. We also indicate the statistical significance of the results. The notation "." indicates a two-sided $10 \%$ significance level, the notation "** stands for a $5 \%$ significance level, "**" indicates a $1 \%$ significance level and "***" represents a $0.1 \%$ significance level.

The regression models (1) and (3) are supported by 7244 observations while the number of observations underlying the results of regression models (4) and (4') lies between 1452 and 3266 depending on the chosen sub-period. We observe an adjusted $R$-squared value above $92 \%$ for all the presented regressions with highly significant effects $(p<0.001)$ of the age and the gender on the prevalence rates. Similar values for the coefficient estimates are found among the considered 
regression models, e.g., the age coefficient for the age class $80-84$ is between 1.333 and 1.423 while the gender coefficient stands between 0.200 and 0.279 . The values taken by the coefficients can be interpreted as follows. A value of 1.374 for the age class 80-84 coefficient (see regression model 1 ) indicates that the prevalence rates multiply by a factor of $\exp (1.374) \approx 3.95$ when compared to the baseline (ages 65-69). In fact, positive coefficient values yield factors larger than one and indicate an increasing effect on the prevalence rates. Negative values (factors below one) signify a decreasing effect. The women's prevalence rates are higher than the men's ones, e.g., we find a gender coefficient of 0.248 meaning that the women's prevalence rates exceed the men's ones by a factor of $\exp (0.248) \approx 1.28$. We investigate on temporal effects through the period variable in the regression models (1) to (4). We observe that the prevalence rates are significantly lower in both periods 1995-2001 (coefficient -0.151) and 2002-2010 (-0.140) when compared to the most recent (baseline) period 2011-2014. The impact of the linguistic regions is first addressed in regression model (1). We note a coefficient of 0.155 and a factor of 0.425 for elderly living in the French (FR) and the Italian (IT) linguistic regions respectively. Based on this result, we conclude that persons living in those two linguistic regions present higher prevalence rates when compared to persons living in the German linguistic region (baseline). Using ZH as the baseline in regression model (3), the results highlight significant cantonal differences, e.g., for the canton VD the prevalence rates exceed those from $\mathrm{ZH}$ by a factor of $\exp (0.218) \approx 1.24$.

Beyond the variables included in the regression model (1), we briefly discuss the question of the age-gender interaction in the following. If adding the term $\sum_{n} \beta_{7}^{n} A G E_{i}^{n} \times G E N D E R_{i}$ in the regression model (1), the coefficients $\beta_{0}, \beta_{1}^{j}, \beta_{4}^{k}$ and $\beta_{5}^{l}$ linked to the intercept, the age, the period and the canton remain significant and their values very close. However, the gender coefficient $\beta_{2}$ yields -0.036 . Thus, we observe that the coefficient $\beta_{2}$ related to the gender changes the sign (from 0.248). At high ages however, there is an important positive contribution to the prevalence rates from the interaction term. The six values of the interaction coefficient $\beta_{7}^{n}$ are $-0.006,0.102,0.300,0.460,0.540$ and 0.604 . In fact, for ages above 75 years the interaction term makes the overall contribution from female to the prevalence rates again positive. This means that women's prevalence rates are higher than the men's ones and increase with ages (Crimmins et al., 2011). The enhanced regression model (2) yields an adjusted $R$-squared value of 0.936 to be compared with 0.924 for the regression model (1). Adding the interaction brings only a small improvement in variance explanation but adds complexity for the interpretation of the coefficients. In the sequel, we do not include further interactions in our model. ${ }^{7}$

We run regression model (4) on the three sub-periods. When only focusing on the differences between the periods, we observe that the gender variable shows a slightly lower coefficient in the period 2011-2014. The year variable presents remarkable differences: the coefficient has a significant effect on the prevalence rates in the first two periods 1995-2001 and 2002-2010, while it has no significant effect in the period 2011-2014. There is no clear interpretation of the year effect on the prevalence rates in the last sub-period. One part of the difference observed through periods comes from the change in law which happened in 2011 (see Section 2.2) but another part might stem from changes in the medical assessment of the severity through the years. Nevertheless, since 2011, prevalence rates seem to be homogeneous in time. In the following sections, we will concentrate on the observations within the period from 2011 to 2014 where the year variable has no effect and the observations are stable over the four years. Therefore, based

\footnotetext{
${ }^{7}$ When adding the age-gender interaction in the forecasts, the robustness analysis in Section 5.3 would yield increased relative errors.
} 
on regression model (4), we define the regression model $\left(4^{\prime}\right)$ which omits the non-significant year variable:

$$
\log \pi_{i}=\beta_{0}+\sum_{j} \beta_{1}^{j} A G E_{i}^{j}+\beta_{2} G E N D E R_{i}+\sum_{m} \beta_{6}^{m} C A N T O N_{i}^{m}+\epsilon_{i}
$$

\subsection{Regression results for the frailty level and the type of care prevalence $\pi^{(s)}$}

In the following, we discuss the results obtained from the application of the regression models (5.s). The specific prevalence rates along the three frailty levels (mild, moderate and severe)

\begin{tabular}{|c|c|c|c|c|c|}
\hline \multirow[t]{2}{*}{ Model } & \multicolumn{3}{|c|}{ Frailty level } & \multicolumn{2}{|c|}{ Type of care } \\
\hline & Mild (5.1) & Moderate (5.2) & Severe $(5.3)$ & At home (5.a) & In an institution (5.b) \\
\hline Intercept & $-5.486 * * *(.056)$ & $-5.415 * * *(.051)$ & $-5.992 * * *(.050)$ & $-5.136 * * *(.057)$ & $-5.324 * * *(.036)$ \\
\hline \multicolumn{6}{|c|}{ Age (baseline: $65-69$ ) } \\
\hline $70-74$ & $-0.084 * \quad(.037)$ & $0.260 * * *(.026)$ & $0.431 * * *(.033)$ & $-0.413 * * *(.037)$ & $0.639 * * *(.024)$ \\
\hline $75-79$ & $0.274 * * *(.037)$ & $0.747 * * *(.026)$ & $1.075 * * *(.033)$ & $-0.440 * * *(.037)$ & $1.334 * * *(.024)$ \\
\hline $80-84$ & $0.719 * * *(.037)$ & $1.467 * * *(.026)$ & $1.725 * * *(.033)$ & $0.205 * * *(.037)$ & $1.957 * * *(.024)$ \\
\hline $85-89$ & $1.149 * * *(.037)$ & $2.082 * * *(.026)$ & 2.336 *** $(.033)$ & 0.706 *** $(.038)$ & $2.552 * * *(.024)$ \\
\hline $90-94$ & $1.463 * * *(.037)$ & $2.595 * * *(.026)$ & $2.904 * * *(.033)$ & $1.053 * * *(.037)$ & $3.095 * * *(.024)$ \\
\hline $95+$ & $1.500 * * *(.039)$ & $2.962 * * *(.026)$ & 3.566 *** $(.033)$ & $1.100 * * *(.039)$ & $3.600 * * *(.024)$ \\
\hline \multicolumn{6}{|c|}{ Gender (baseline: Male) } \\
\hline Female & $0.150 * * *(.020)$ & $0.141 * * *(.010)$ & $0.244 * * *(.017)$ & $0.124 * * *(.020)$ & $0.207 * * *(.013)$ \\
\hline \multicolumn{6}{|c|}{ Canton (baseline: $\mathrm{ZH}$ ) } \\
\hline $\mathrm{AG}$ & 0.138 & $(.050)$ & $0.088 \quad(.063)$ & $0.174 *$ & $(.045)$ \\
\hline $\mathrm{AI}$ & $(.077)$ & $(.052)$ & $0.311 * * *(.064)$ & 0.130 & $0.107 *$ \\
\hline $\mathrm{AR}$ & $-0.168 * \quad(.072)$ & -0.063 & $-0.003 \quad(.063)$ & $-0.171 *$ & -0.012 \\
\hline $\mathrm{BE}$ & $0.211 * * \quad(.070)$ & $-0.091 . \quad(.050)$ & 0.018 & $0.193 * * \quad(.072)$ & -0.018 \\
\hline $\mathrm{BL}$ & $0.208 * * \quad(.070)$ & $0.204 * * *(.050)$ & $0.105 . \quad(.063)$ & $0.287 * * *(.072)$ & $0.132 * * \quad(.045)$ \\
\hline $\mathrm{BS}$ & $0.407 * * *(.071)$ & $0.275 * * *(.050)$ & $0.344 * * *(.063)$ & 0.431 *** $(.072)$ & $0.307 * * *(.045)$ \\
\hline FR & $0.026 \quad(.071)$ & $0.348 * * *(.050)$ & 0.430 *** (.063) & $0.112 \quad(.072)$ & $0.357 * * *(.045)$ \\
\hline GE & $0.390 * * *(.070)$ & $-0.115 * \quad(.050)$ & $0.181 * * \quad(.063)$ & $0.472 * * *(.072)$ & $0.008 \quad(.045)$ \\
\hline GL & $-0.048 \quad(.072)$ & $-0.225 * * *(.051)$ & $-0.131 * \quad(.063)$ & $-0.163 * \quad(.074)$ & -0.082 \\
\hline GR & $0.155 * \quad(.071)$ & $-0.197 * * *(.050)$ & $-0.222 * * *(.063)$ & $0.191 * * \quad(.072)$ & $-0.184 * * *(.045)$ \\
\hline $\mathrm{JU}$ & $0.661 * * *(.071)$ & $0.304 * * *(.050)$ & 0.336 *** (.063) & 0.691 *** (.072) & $0.291 * * *(.045)$ \\
\hline $\mathrm{LU}$ & $0.285 * * *(.071)$ & $0.364 * * *(.050)$ & $0.417 * * *(.063)$ & $0.363 * * *(.072)$ & $0.355 * * *(.045)$ \\
\hline $\mathrm{NE}$ & $0.330 * * *(.070)$ & $-0.397 * * *(.050)$ & $0.187 * * \quad(.063)$ & $0.459 * * *(.072)$ & $-0.131 * * \quad(.045)$ \\
\hline NW & $0.223 * * \quad(.072)$ & $0.172 * * *(.050)$ & $-0.160 * \quad(.063)$ & $0.402 * * *(.073)$ & $0.005 \quad(.045)$ \\
\hline OW & $0.171 * \quad(.071)$ & $0.126 * \quad(.050)$ & 0.046 & 0.309 *** (.074) & $0.091 *$ \\
\hline SG & $-0.008 \quad(.070)$ & $0.009 \quad(.050)$ & $0.087 \quad(.063)$ & $0.023 \quad(.072)$ & $0.041 \quad(.045)$ \\
\hline $\mathrm{SH}$ & $0.215 * * \quad(.070)$ & $-0.289 * * *(.036)$ & $-0.229 * * *(.063)$ & 0.274 *** $(.072)$ & $-0.266 * * *(.045)$ \\
\hline $\mathrm{SO}$ & $0.399 * * *(.070)$ & $0.277 * * *(.050)$ & $0.159 * \quad(.063)$ & $0.463 * * *(.072)$ & $0.215 * * *(.045)$ \\
\hline $\mathrm{SZ}$ & $0.059 \quad(.072)$ & $0.018 \quad(.050)$ & 0.244 *** $(.063)$ & $0.075 \quad(.073)$ & $0.137 * * \quad(.045)$ \\
\hline $\mathrm{TG}$ & -0.103 & $-0.159 * * \quad(.050)$ & $-0.207 * * \quad(.063)$ & -0.028 & $-0.209 * * *(.045)$ \\
\hline $\mathrm{TI}$ & -0.134 & $0.527 * * *(.050)$ & $0.820 * * *(.063)$ & -0.018 & $0.620 * * *(.045)$ \\
\hline UR & $-0.054 \quad(.072)$ & $0.060 \quad(.050)$ & $0.227 * * *(.063)$ & 0.058 & $0.131 * * \quad(.045)$ \\
\hline VD & $0.309 * * *(.070)$ & $0.191 * * *(.050)$ & $0.365 * * *(.063)$ & $0.388 * * *(.072)$ & $0.258 * * *(.045)$ \\
\hline VS & $0.530 * * *(.070)$ & 0.352 *** (.050) & 0.551 *** $(.063)$ & 0.566 *** $(.072)$ & $0.443 * * *(.045)$ \\
\hline $\mathrm{ZG}$ & $-0.324 * * *(.071)$ & $0.120 * \quad(.050)$ & $0.002 \quad(.063)$ & $-0.268 * * *(.073)$ & $0.081 . \quad(.045)$ \\
\hline$N$ & 1401 & 1442 & 1450 & 1402 & 1450 \\
\hline Adj. $R^{2}$ & 0.767 & 0.945 & 0.933 & 0.744 & 0.963 \\
\hline
\end{tabular}

Note: $. p<0.1,{ }^{*} p<0.05,{ }^{* *} p<0.01,{ }^{* * *} p<0.001$.

Table 7: Results for the regression models (5.s) along the frailty levels and the types of care for the years from 2011 to 2014 . 
and the two types of care (at home and in an institution) are considered for dependent variables. The results allow to take a closer look at the differences in the rates between the frailty levels and the types of care. As mentioned above, we solely consider observations from the period 2011-2014.

The results presented in Table 7 are statistically significant for most of the explanatory variables, i.e., the age, the gender and the canton of residence are critical factors in determining the prevalence rates along the various acuity levels. For the age class coefficients, we find again a positive relation between the age and the prevalence rate when compared to the baseline 65-69. This means that older population segments come along with higher prevalence rates. In the frailty level regression models (5.1) to (5.3), the age effect is more important for the moderately and the severely dependent persons than for the mildly ones since we observe higher values for the coefficients through all age classes. We find a similar situation in the type of care models (5.a) and (5.b), in which the age has a higher effect on the prevalence rates of the elderly cared for in an institution than of those cared for at home. We also observe differences in the gender effect. The positive sign of the coefficient underlines the idea that females show higher prevalence rates than males, particularly for severely dependent persons and persons cared for in an institution. This gender difference finds an explanation in the life expectancy with women living longer than men (FSO, 2017). The last factor regards the region of residence and compares the prevalence rates for individuals living in one of the cantons other than Zurich $(\mathrm{ZH})$ to the ones from ZH. When considering some of the main cantons in term of inhabitants, e.g., Bern (BE), Basel (BS), Geneva (GE), Ticino (TI) and Vaud (VD), we observe for some acuity levels significant differences in the coefficients when compared to ZH. For severely dependent elderly, the statistically significant coefficients are positive for all the above cantons. This comment also holds for persons receiving care either at home or in an institution. However, the moderately dependent elderly show a more heterogeneous situation with negative coefficients for $\mathrm{BE}$ and GE.

\subsection{Discussion on the robustness of the results}

In this part, we aim to assess the models and the robustness of our results. We first verify the homoscedasticity and the OLS normality hypothesis of residuals. Then, on the example of the regression models $\left(4^{\prime}\right)$ and (5.1) to (5.3), we check the robustness of the estimates by bootstrapping the confidence intervals of the regression coefficients.

\section{OLS hypothesis tests}

Since we produce prevalence rates forecasts based on the regression models $\left(4^{\prime}\right)$ and $(5 . s)$ in Section 5, we discuss the hypothesis under which residuals are homoscedastic and normally distributed. In comparison to the heteroscedasticity assumption, the homoscedasticity assumption ensures having both a well specified regression model and not missing any important explanatory variables (Manning, 1998; Manning and Mullahy, 2001). Further, the normality assumption has some statistical importance when defining the correct approach for determining confidence intervals and statistical tests.

We first test the homoscedasticity assumption by undergoing three tests: the Non-Constant Error Variance (NCV) test (Cook and Weisberg, 1983), the Breush-Pagan test (Trevor and Adrian, 1979) and a directional test present in the gvlma R package (Ohri, 2012). Both the NCV 
and the Breush-Pagan tests reject the hypothesis under which the residuals are homoscedastic while the directional test does not reject it. Therefore, we might be facing a situation of heteroscedasticity. Usual methods for solving heteroscedasticity consider adding independent variables to the model or reformulating the linear regression model. Since our dataset does not contain other variables we are not able to clarify this problem by adding other dependent variables. Nonetheless, we statistically support the choice of a log-linear specification due to the absence of dependence structure in the plot of the regression residuals. In Figure 5, we illustrate the residuals for the regression models $\left(4^{\prime}\right)$ and (5.1) to (5.3).

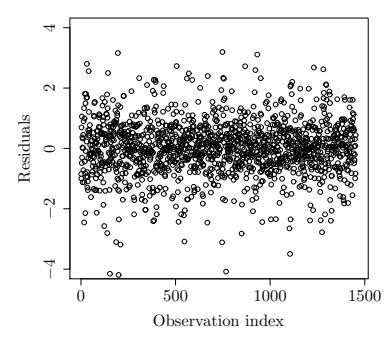

$\left(4^{\prime}\right)$

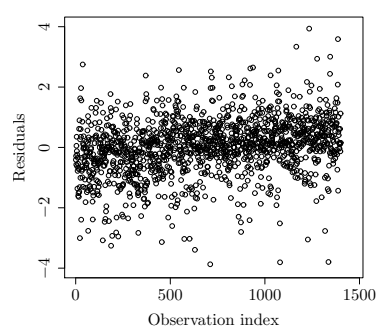

$(5.1)$

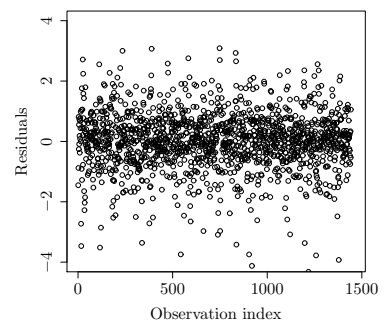

$(5.2)$

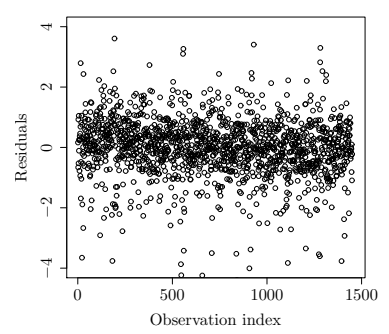

$(5.3)$

Figure 5: Residuals for the regression models $\left(4^{\prime}\right)$ and (5.1) to (5.3).

Thereafter, we test the normality assumption by first using the Kolmogorov-Smirnov normality test (Lopes, 2011). For the regression models $\left(4^{\prime}\right)$ and (5.1) to (5.3), the results reject the normality assumption with $p$-values below 0.01 . Secondly, the quantile-quantile plots presented in Figure 6 report the empirical standardized residuals against the theoretical quantiles from the normal distribution. The values within the $95 \%$-confidence interval $(-1.96,1.96)$ appear close to normal while the tails are rather non-normal. Finally, following Lumley et al. (2002), linear regressions are valid in large samples for any distribution. In our case, the regression models $\left(4^{\prime}\right)$ and (5.s) are supported by more than 1400 data points (cf. the number $N$ in Tables 6 and 7) which should well ensure the applicability of the central limit theorem and validate the regression results.

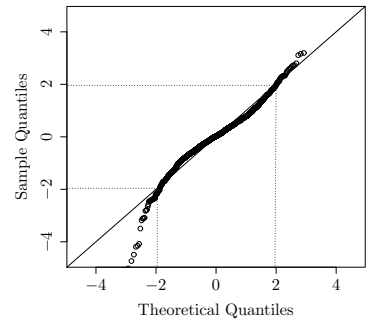

$\left(4^{\prime}\right)$

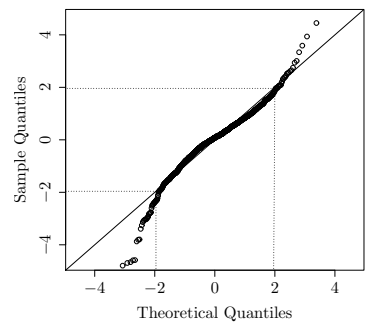

$(5.1)$

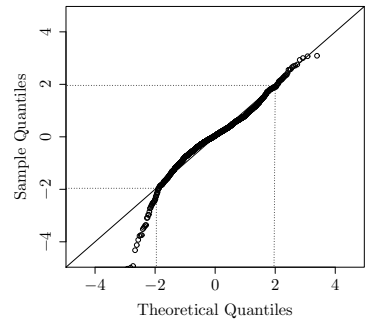

$(5.2)$

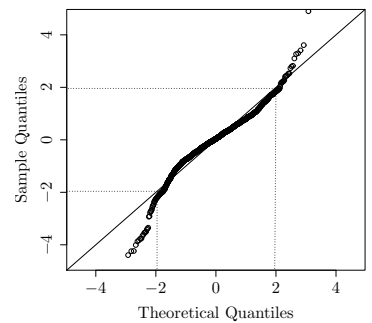

$(5.3)$

Figure 6: QQ-plots of the residuals for the regression models $\left(4^{\prime}\right)$ and (5.1) to (5.3).

\section{Bootstrapping of regression coefficients}

In our study, we use the non-parametric bootstrap method for estimating the 95\%-confidence bounds for the OLS coefficients on samples drawn from the same population. The nonparametric bootstrap method (Efron, 1979; Efron and Tibshirani, 1993) consists in drawing 
many samples from the original population and computing the distribution statistics. Each sample corresponds to a re-sampling with replacement of the original dataset with equal size, i.e., observations from the original dataset can be eliminated or replicated in a bootstrap dataset. We define $S_{\mathcal{B}}$ the number of bootstrap samples and $N_{\mathcal{B}}$ the size of each sample. To ensure convergence and stability in the statistics we use $S_{\mathcal{B}}=10000$ bootstrap samples. We take the size $N_{\mathcal{B}}$ equal to the original number of underlying observations. The analysis of the $2.5 \%$ and $97.5 \%$-quantiles is an alternative to the $p$-value for assessing the significance of a regression coefficient. Highly significant results will display tight confidence intervals while wider intervals or sign changes highlight non-significant outcomes.

\begin{tabular}{|c|c|c|c|c|c|c|c|c|}
\hline \multirow[t]{2}{*}{ Model } & \multicolumn{2}{|c|}{$\left(4^{\prime}\right)$} & \multicolumn{2}{|c|}{$(5.1)$} & \multicolumn{2}{|c|}{$(5.2)$} & \multicolumn{2}{|c|}{$(5.3)$} \\
\hline & $q_{2.5 \%}$ & $q_{97.5 \%}$ & $q_{2.5 \%}$ & $q_{97.5 \%}$ & $q_{2.5 \%}$ & $q_{97.5 \%}$ & $q_{2.5 \%}$ & $q_{97.5 \%}$ \\
\hline Intercept & -4.557 & -4.466 & -5.564 & -5.416 & -5.464 & -5.366 & -6.065 & -5.922 \\
\hline \multicolumn{9}{|c|}{ Age (baseline: 65-69) } \\
\hline $70-74$ & 0.151 & 0.238 & -0.155 & -0.014 & 0.204 & 0.315 & 0.361 & 0.500 \\
\hline $75-79$ & 0.663 & 0.741 & 0.213 & 0.336 & 0.693 & 0.801 & 1.010 & 1.139 \\
\hline $80-84$ & 1.296 & 1.370 & 0.659 & 0.778 & 1.420 & 1.515 & 1.664 & 1.785 \\
\hline $85-89$ & 1.870 & 1.944 & 1.085 & 1.213 & 2.035 & 2.128 & 2.276 & 2.395 \\
\hline $90-94$ & 2.387 & 2.469 & 1.395 & 1.532 & 2.542 & 2.648 & 2.835 & 2.973 \\
\hline $95+$ & 2.821 & 2.917 & 1.403 & 1.597 & 2.902 & 3.020 & 3.488 & 3.644 \\
\hline \multicolumn{9}{|c|}{ Gender (baseline: Male) } \\
\hline Female & 0.179 & 0.220 & 0.111 & 0.188 & 0.114 & 0.168 & 0.210 & 0.278 \\
\hline \multicolumn{9}{|c|}{ Canton (baseline: ZH) } \\
\hline $\mathrm{AG}$ & 0.035 & 0.133 & 0.038 & 0.236 & -0.018 & 0.095 & 0.014 & 0.165 \\
\hline $\mathrm{AI}$ & -0.043 & 0.220 & -0.154 & 0.212 & -0.065 & 0.183 & 0.138 & 0.491 \\
\hline $\mathrm{AR}$ & -0.144 & 0.023 & -0.312 & -0.022 & -0.167 & 0.038 & -0.101 & 0.099 \\
\hline $\mathrm{BE}$ & -0.023 & 0.061 & 0.134 & 0.292 & -0.136 & -0.045 & -0.050 & 0.086 \\
\hline BL & 0.130 & 0.219 & 0.117 & 0.302 & 0.152 & 0.253 & 0.020 & 0.189 \\
\hline BS & 0.268 & 0.388 & 0.312 & 0.503 & 0.213 & 0.334 & 0.258 & 0.431 \\
\hline FR & 0.282 & 0.375 & -0.075 & 0.128 & 0.293 & 0.402 & 0.354 & 0.504 \\
\hline GE & 0.067 & 0.149 & 0.299 & 0.486 & -0.169 & -0.060 & 0.107 & 0.256 \\
\hline GL & -0.175 & -0.039 & -0.175 & 0.085 & -0.366 & -0.090 & -0.271 & 0.002 \\
\hline GR & -0.183 & -0.073 & 0.058 & 0.256 & -0.270 & -0.130 & -0.321 & -0.122 \\
\hline JU & 0.338 & 0.476 & 0.541 & 0.776 & 0.218 & 0.382 & 0.227 & 0.443 \\
\hline $\mathrm{LU}$ & 0.321 & 0.411 & 0.191 & 0.381 & 0.316 & 0.411 & 0.345 & 0.489 \\
\hline $\mathrm{NE}$ & -0.043 & 0.062 & 0.215 & 0.449 & -0.495 & -0.306 & 0.083 & 0.287 \\
\hline NW & 0.051 & 0.211 & 0.064 & 0.379 & 0.060 & 0.278 & -0.338 & 0.009 \\
\hline OW & 0.068 & 0.228 & -0.015 & 0.350 & 0.013 & 0.229 & -0.102 & 0.191 \\
\hline $\mathrm{SG}$ & -0.012 & 0.081 & -0.087 & 0.074 & -0.036 & 0.054 & 0.003 & 0.169 \\
\hline $\mathrm{SH}$ & -0.202 & -0.070 & 0.080 & 0.352 & -0.387 & -0.193 & -0.332 & -0.126 \\
\hline $\mathrm{SO}$ & 0.236 & 0.315 & 0.296 & 0.508 & 0.222 & 0.330 & 0.081 & 0.239 \\
\hline $\mathrm{SZ}$ & 0.075 & 0.172 & -0.055 & 0.172 & -0.050 & 0.086 & 0.162 & 0.325 \\
\hline $\mathrm{TG}$ & -0.205 & -0.099 & -0.202 & -0.005 & -0.225 & -0.093 & -0.295 & -0.120 \\
\hline $\mathrm{TI}$ & 0.508 & 0.614 & -0.270 & -0.007 & 0.466 & 0.583 & 0.742 & 0.896 \\
\hline UR & 0.063 & 0.190 & -0.195 & 0.086 & -0.036 & 0.152 & 0.091 & 0.353 \\
\hline VD & 0.235 & 0.323 & 0.227 & 0.394 & 0.140 & 0.241 & 0.283 & 0.445 \\
\hline VS & 0.419 & 0.533 & 0.443 & 0.620 & 0.300 & 0.403 & 0.453 & 0.645 \\
\hline $\mathrm{ZG}$ & -0.029 & 0.089 & -0.459 & -0.186 & 0.035 & 0.200 & -0.119 & 0.117 \\
\hline$S_{\mathcal{B}}$ & 10000 & & 10000 & & 10000 & & 10000 & \\
\hline$N_{\mathcal{B}}$ & 1452 & & 1401 & & 1442 & & 1450 & \\
\hline
\end{tabular}

Table 8: 95\%-confidence bounds from the bootstrap method on the coefficients of the regression models $\left(4^{\prime}\right)$ and (5.1) to (5.3). 
Table 8 presents the $2.5 \%$ - and $97.5 \%$-quantiles $\left(q_{2.5 \%}\right.$ and $\left.q_{97.5 \%}\right)$ of the $\beta$ coefficients' estimates for the regression models $\left(4^{\prime}\right)$ and (5.1) to (5.3). We compare the bootstrap outcomes with the estimates presented in Table 7 . The confidence bounds for the intercept and the age coefficients are tight. For example, in the model $\left(4^{\prime}\right)$ and for the gender coefficient, the 95\%-confidence bounds $q_{2.5 \%}=0.179$ and $q_{97.5 \%}=0.220$ closely surround the coefficient estimate of 0.200 (see Table 6). Over the three frailty level models (5.1) to (5.3), we observe slightly wider confidence intervals for the gender coefficient estimates. In model (5.1) we find $q_{2.5 \%}=0.111$ and $q_{97.5 \%}=0.188$ for a coefficient estimate of 0.150 (see Table 7 ). In the analysis of the results for the cantons' coefficients, we observe typical patterns that support our models. Only the regression coefficients showing statistically non-significant estimates (see Tables 6 and 7) have wider confidence intervals and present sign changes between the lower and upper confidence bounds. This is the case for the cantons of AR, BE, NE, SG and ZG in regression model $\left(4^{\prime}\right)$ the cantons of AI, FR, GL, OW, SG, SZ and UR in regression model (5.1), the cantons of AG, AI, AR, SG, SZ and UR in model (5.2) and the cantons of AR, BE, GL, NW, OW and ZG in model (5.3) where the reported coefficients' estimates are non-significant. Some cantons like AI in model (4'), OW in model (5.1) and GL and NW in model (5.3) present sign changes in the confidence intervals obtained from the bootstrap method although the regression coefficients are statistically significant at level $p<0.05$.

\section{Forecast of the dependent population until 2045}

In the sequel, departing from the historical prevalence rate data, we produce forecasts for the years from 2015 to 2045 . We first describe the methodology and define an index that simplifies the comparison between years. Our predictions will be based on the regression models introduced in Sections 3 and 4 taking into account regional differences along with the age and the gender parameters (model $4^{\prime}$ ) and considering the acuity levels (models 5.s). We put the development of the prevalence rates in the historical context by reporting the values for the years before 2015 .

\subsection{Forecast methodology and demographic scenarios}

From the regression models $\left(4^{\prime}\right)$ and (5.s) applied on the period 2011-2014, we define the predicted prevalence rates $\hat{\pi}_{i}$ and $\hat{\pi}_{i}^{(s)}$ as follows (see Stock and Watson, 2012):

$$
\hat{\pi}_{i}=\exp \left[\hat{\beta}_{0}+\sum_{j} \hat{\beta}_{1}^{j} A G E_{i}^{j}+\hat{\beta}_{2} G E N D E R_{i}+\sum_{m} \hat{\beta}_{6}{ }^{m} C A N T O N_{i}^{m}\right] \times \overline{\exp \left(\hat{\epsilon_{i}}\right)} \equiv \hat{\pi}_{A, G, C},
$$

and

$$
\hat{\pi}_{i}^{(s)}=\exp \left[\hat{\beta}_{0}+\sum_{j} \hat{\beta}_{1}^{j} A G E_{i}^{j}+\hat{\beta}_{2} G E N D E R_{i}+\sum_{m} \hat{\beta}_{6}^{m} C A N T O N_{i}^{m}\right] \times \overline{\exp \left(\hat{\epsilon_{i}}\right)} \equiv \hat{\pi}_{A, G, C}^{(s)}
$$

where $s \in\{1,2,3\}$ or $\{\mathrm{a}, \mathrm{b}\}$ depending if the frailty levels or the types of care are in the focus. We denote with a hat "^" the $\beta$ coefficient estimates and the estimated prevalence rates. In the above Equations $(6)$ and $(6 . s)$, the term $\overline{\exp \left(\hat{\epsilon_{i}}\right)}$ stands for the exponential residuals sample mean. In the sequel, we prefer the notation $\hat{\pi}_{A, G, C}$ to the notation $\hat{\pi}_{i}$ where the indexes $A, G$ and $C$ are a short hand notation for the age class, the gender and the canton variables. In the further description below, we omit the $(s)$ in the notations since the calculations apply in the 
same way for each $\hat{\pi}_{A, G, C}^{(s)}$ than for $\hat{\pi}_{A, G, C}$.

We use the predictions to determine the future number of dependent persons $\hat{\mathcal{N}}_{t, A, G, C}$ per age $A$, gender $G$ and canton $C$ in the years $t \geq 2015$ by multiplying the predicted (time-independent, cf. Section 4.2) prevalence rate $\hat{\pi}_{A, G, C}$ with the corresponding underlying population forecast $\hat{\mathcal{P}}_{t, A, G, C}$ :

$$
\hat{\mathcal{N}}_{t, A, G, C}=\hat{\pi}_{A, G, C} \times \hat{\mathcal{P}}_{t, A, G, C} .
$$

Thereby, the population forecast $\hat{\mathcal{P}}_{t, A, G, C}$ is taken from the FSO (see also Figure 4 and Tables 2 and 3 in Section 2.4). The choice of the demographic scenario has an important influence on the results (Costa-Font et al., 2008). Therefore, on the one hand, we base our analysis on the reference scenario (Section 5.2), and, on the other hand, we consider high and low scenarios for studying sensitivities (Section 5.3). Our predictions are based on the assumption that the relative structure of the dependence rates will remain the same in the future. Other approaches consider improvement in the old-age prevalence in the future (e.g., European Commission, 2015).

For reporting the results in Section 5.2 (see Tables 9 and 10), we introduce the dependent population prevalence index $\hat{I}_{t}$ corresponding to the ratio between the aggregate dependent population forecast over all ages and both genders $\hat{\mathcal{N}}_{t, C} \equiv \sum_{A, G} \hat{\mathcal{N}}_{t, A, G}$ for each canton $C$ in year $t$ and the corresponding value $\mathcal{N}_{2014, C}$ in the year 2014 as follows: ${ }^{8}$

$$
\hat{I}_{t, C}=\frac{\hat{\mathcal{N}}_{t, C}}{\mathcal{N}_{2014, C}}
$$

Thus, by definition $I_{2014}=1$. Similarly, we calculate the corresponding index for the three language regions and for the whole of Switzerland by summing the population numbers over the respective cantons. The index is calculated following the same logic for the dependent population by acuity levels, see Table 10. It is also interesting to study the relative weight of the dependent population in the various cantons and language regions. We derive the dependent population share forecast $\hat{S}_{t, C}$ for each canton $C$ in year $t$ as the ratio between $\hat{\mathcal{N}}_{t, C}$ and the total population forecast $\hat{\mathcal{N}}_{t} \equiv \sum_{C} \hat{\mathcal{N}}_{t, C}$ :

$$
\hat{S}_{t, C}=\frac{\hat{\mathcal{N}}_{t, C}}{\hat{\mathcal{N}}_{t}}
$$

The aggregate shares at the level of the linguistic regions are derived in the same way.

\section{$5.2 \quad$ Forecasts}

In Table 9, we present the evolution of the number of dependent elderly by year, canton and linguistic region over the period 1995 to 2045. The results for the years before and including 2014 are based on historical values while the ones from 2015 are forecasts based on the reference scenario. We present the index $\hat{I}_{t}$ and the share $\hat{S}_{t}$ in parenthesis as defined in the Equations (9) and (10). In the following discussion, we do not comment changes in dependent population shares since they remain relatively stable through the years for the major part of the cantons.

\footnotetext{
${ }^{8}$ For years before 2014, we omit the hat "^” in our notation since the numbers are reported statistics and do not represent forecast estimates.
} 


\begin{tabular}{|c|c|c|c|c|c|c|c|c|c|c|c|c|c|c|}
\hline \multirow[t]{2}{*}{ Year $t$} & \multicolumn{2}{|c|}{1995} & \multicolumn{2}{|c|}{2005} & \multicolumn{2}{|c|}{2014} & \multicolumn{2}{|c|}{2015} & \multicolumn{2}{|c|}{2025} & \multicolumn{2}{|c|}{2035} & \multicolumn{2}{|c|}{2045} \\
\hline & $I_{t, C}$ & $S_{t, C}$ & $I_{t, C}$ & $S_{t, C}$ & $I_{t, C}$ & $S_{t, C}$ & $\hat{I}_{t, C}$ & $\hat{S}_{t, C}$ & $\hat{I}_{t, C}$ & $\hat{S}_{t, C}$ & $\hat{I}_{t, C}$ & $\hat{S}_{t, C}$ & $\hat{I}_{t, C}$ & $\hat{S}_{t, C}$ \\
\hline \multicolumn{15}{|c|}{ Cantons } \\
\hline $\mathrm{AG}$ & 0.52 & $(5.87)$ & 0.67 & $(5.98)$ & 1.00 & $(6.43)$ & 1.02 & $(6.37)$ & 1.47 & $(6.73)$ & 2.13 & $(7.13)$ & 2.85 & $(7.45)$ \\
\hline $\mathrm{AI}$ & 0.77 & $(0.25)$ & 0.88 & $(0.22)$ & 1.00 & $(0.18)$ & 1.04 & $(0.18)$ & 1.43 & & 1.92 & & 2.44 & \\
\hline $\mathrm{AR}$ & 0.77 & $(0.77)$ & 0.90 & $(0.70)$ & 1.00 & $(0.57)$ & 1.04 & $(0.57)$ & 1.36 & $(0.55)$ & 1.81 & & 2.27 & $(0.52)$ \\
\hline $\mathrm{BE}$ & 0.77 & $(16.22)$ & 0.86 & (14.19) & 1.00 & (12.04) & 1.04 & (12.16) & 1.38 & (11.86) & 1.87 & (11.75) & 2.34 & (11.49) \\
\hline $\mathrm{BL}$ & 0.51 & $(3.50)$ & 0.70 & $(3.77)$ & 1.00 & $(3.95)$ & 1.04 & $(3.97)$ & 1.41 & $(3.9$ & 1.85 & $(3.80)$ & 2.24 & $(3.60)$ \\
\hline $\mathrm{BS}$ & 0.65 & $(4.15$ & 0.73 & (3.6 & 1.00 & (3.65) & 1.02 & $(3.66)$ & 1.16 & & 1.35 & & 1.60 & $(2.40)$ \\
\hline FR & 0.59 & $(3.33)$ & 0.88 & $(3.86)$ & 1.00 & $(3.21)$ & 1.09 & $(3.40)$ & 1.53 & $(3.5$ & 2.25 & & 3.04 & $(3$. \\
\hline GE & 0.50 & $(4.65)$ & 0.62 & $(4.5$ & 1.00 & $(5.34)$ & 1.03 & & 1.38 & & 1.81 & & 2.23 & \\
\hline GL & 0.92 & $(0.64)$ & 1.00 & $(0.55)$ & 1.00 & $(0.4$ & 1.04 & & 1.36 & & 1.84 & & 2.38 & \\
\hline GR & 0.77 & $(2.5$ & 0.92 & $(2.41)$ & 1.00 & $(1.9$ & 1.03 & & 1.42 & & 1.97 & & 2.48 & \\
\hline $\mathrm{JU}$ & 0.74 & $(1.6 !$ & 0.74 & (1. & 1.00 & & 1.01 & & 1.34 & & 1.79 & & 2.26 & \\
\hline $\mathrm{LU}$ & 0.56 & $(5.40)$ & 0.71 & $(5.30$ & 1.00 & $(5.4$ & 1.05 & $(5$. & 1.42 & $(5$. & 1.93 & & 2.57 & (5. \\
\hline $\mathrm{NE}$ & 0.78 & $(2.5$ & 0.85 & $(2$. & 1.00 & (1. & 1.10 & $(2$. & 1.36 & & 1.75 & & 2.14 & \\
\hline NW & 0.3 & $(0.3$ & 0.51 & $(0$. & 1.00 & $(0.5$ & 0.98 & & 1.53 & & 2.22 & & 2.73 & \\
\hline OW & 0.51 & (0.3 & 0.75 & $(0.4$ & 1.00 & $(0.4$ & 1.00 & $(0$. & 1.53 & & 2.29 & & 3.03 & \\
\hline $\mathrm{SG}$ & 0.60 & $(5.2$ ? & 0.78 & (5. & 1.00 & (5. & 1.02 & & 1.41 & & 1.95 & & 2.52 & \\
\hline $\mathrm{SH}$ & 0.83 & $(1.18$ & 0.96 & $(1.06)$ & 1.00 & $(0.8$ & 1.07 & $(0$. & 1.44 & & 1.93 & & 2.44 & \\
\hline $\mathrm{SO}$ & 0.44 & & & & & & 1.02 & & 1.40 & & 1.90 & & 2.44 & \\
\hline $\mathrm{SZ}$ & 0.56 & $(1.49)$ & 0.70 & (1.4 & 1.00 & (1.5 & 1.04 & & 1.56 & & 2.30 & & 3.14 & \\
\hline TG & 0.64 & $(2.34)$ & 0.88 & $(2.5$ & 1.00 & (2.0 & 1.04 & $(2.1$ & 1.53 & & 2.25 & & 3.06 & $(2$. \\
\hline $\mathrm{TI}$ & 0.41 & $(6.01$ & 0.74 & (8.3 & 1.00 & (8.3 & 0.99 & (8.0 & 1.41 & & 1.91 & & 2.38 & $(8$. \\
\hline UR & 0.69 & $(0.58$ & 1.01 & $(0.67)$ & 1.00 & $(0.4$ & 0.99 & $(0.4$ & 1.36 & & 1.89 & & 2.41 & $(0.47)$ \\
\hline VD & 0.53 & & .63 & & 1.00 & & 1.03 & & 1.37 & & 1.89 & & 2.46 & $(9.85)$ \\
\hline VS & 0.40 & $(3.96)$ & 0.58 & $(4.49)$ & 1.00 & $(5.64)$ & 0.99 & $(5.4$ & 1.43 & & 2.06 & & 2.68 & $(6.18)$ \\
\hline $\mathrm{ZG}$ & 0.38 & & 0.54 & & 1.00 & & 1.02 & & 1.53 & & 2.19 & & 2.85 & $(1.25)$ \\
\hline $\mathrm{ZH}$ & 0.57 & $(14.07)$ & 0.70 & (13.63) & 1.00 & (14.13) & 1.03 & (14.13) & 1.37 & $(13.84)$ & 1.84 & $(13.5$ & 2.33 & $(13.40)$ \\
\hline \multicolumn{15}{|c|}{ Linguistic regions } \\
\hline $\mathrm{DE}$ & 0.61 & $(68.71)$ & 0.75 & $(66.73)$ & 1.00 & $(64.56)$ & 1.03 & $(64.67)$ & 1.40 & & 1.91 & & 2.44 & $(64.20)$ \\
\hline FR & 0.53 & $(25.28)$ & 0.67 & $(24.88)$ & 1.00 & $(27.14)$ & 1.03 & $(27.29)$ & 1.40 & $(27.2$ & 1.94 & $(27.48)$ & 2.50 & $(27.70)$ \\
\hline $\mathrm{IT}$ & 0.41 & $(6.01)$ & 0.74 & $(8.39)$ & 1.00 & $(8.30)$ & 0.99 & $(8.04)$ & 1.41 & $(8.39)$ & 1.91 & $(8.32)$ & 2.38 & $(8.10)$ \\
\hline \multicolumn{15}{|c|}{ Whole country } \\
\hline $\mathrm{CH}$ & 0.57 & $(100.00)$ & 0.73 & $(100.00)$ & 1.00 & $100.00)$ & 1.03 & $(100.00)$ & 1.40 & $(100.00)$ & 1.92 & $(100.00)$ & 2.45 & $(100.00)$ \\
\hline
\end{tabular}

In gray shadow: Index $2014=1.00$.

Table 9: Evolution of the dependent population by cantons in the reference population scenario.

Regarding the development of LTC needs, we can cluster the cantons in different groups. The large cantons of $\mathrm{BE}, \mathrm{TI}, \mathrm{VD}, \mathrm{ZH}$ are the cantons with the highest number of dependent elderly and together cover more than $40 \%$ of the total dependent population. In these four cantons, our results predict that the dependent population in need of LTC will close to double by 2035 in comparison to the 2014 population and be multiplied by about 2.4 by 2045 . Considering the cantons of AG, LU, NW, SG, VS and ZG we observe a slightly higher development of the population in need of care compared to the previous four cantons. We expect their dependent population to be multiplied by two until 2035 and by approximately 2.6 in 2045 . Thereafter, the predictions for the cantons of FR, OW, SZ and TG describe the dependent population to more than triple in 2045. Finally, we can cluster the cantons showing predictions slightly below the average. They are the cantons of BL, GE, JU and NE. For example, at the 2045 time horizon, the dependent population is predicted to increase only by a factor 2.23 for GE. We also highlight the particular case of BS which presents the lowest increase, i.e., the dependent population would only increase by a factor of 1.60 by 2045 when compared to 2014 . 
At the bottom of Table 9, we analyze the evolution in the dependent population when the cantons are clustered by linguistic regions. The shares presented in parenthesis demonstrate that the German-speaking region (DE) covers more than sixty percent of the elderly dependent population whereas around twenty-five percent are located in the French-speaking region (FR) and less than ten percent in the Italian-speaking region (IT). Our predictions do not reveal significant differences in the evolution of the elderly in need of LTC living in the German- or the French-speaking regions. Over the 30-year time frame, the results forecast an increase by a factor of 2.44 and 2.50 in the dependent population of the German and French linguistic regions, respectively. Focusing on the Italian-speaking region, we denote a change of slightly smaller range, i.e., the factor of increase is 2.38 which compares to the factors 2.44 and 2.50 discussed above.

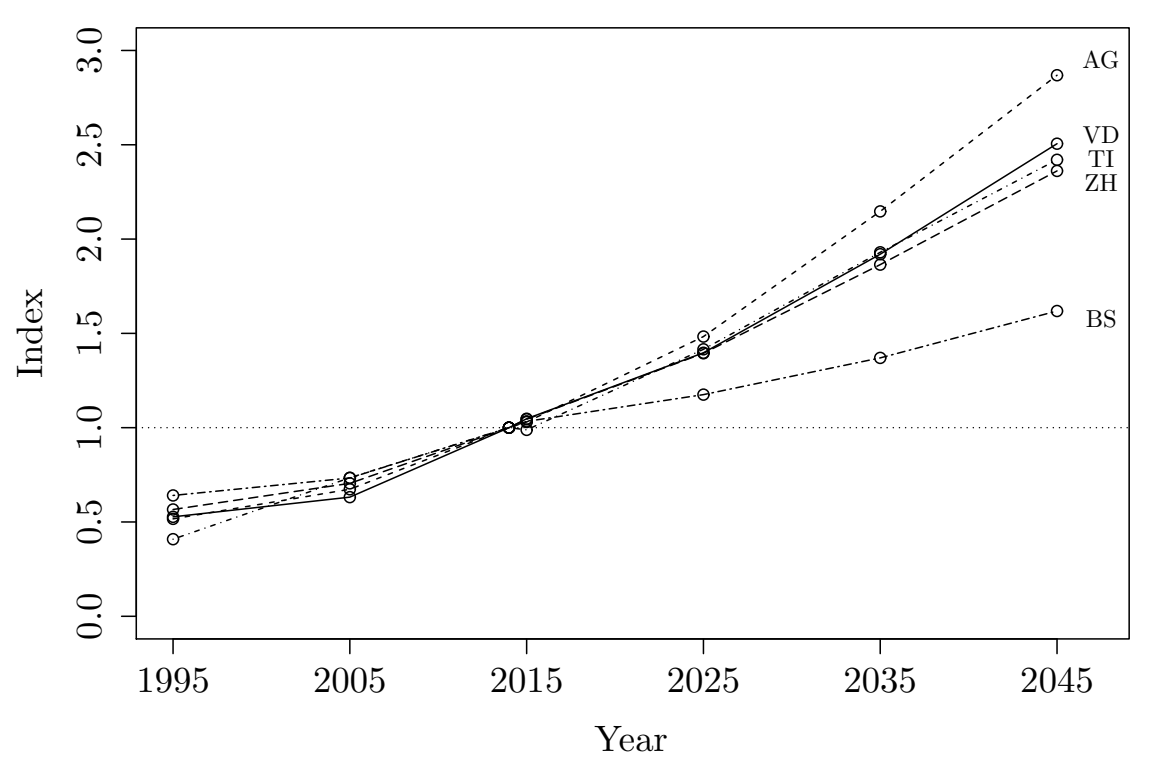

Figure 7: Evolution of the dependent population in five selected cantons in the reference population scenario.

Figure 7 presents a graphical illustration of the indexes for some of the largest cantons (ZH, VD, TI) in the three linguistic regions over the period 1995 to 2045 . As noted in the previous discussion, we denote a common pattern in the evolution of the population for the cantons of Zurich (ZH), Vaud (VD) and Ticino (TI). In the illustration, we also display the predictions for the cantons of AG and of BS differing from the others by their age pyramid and urbanization level.

Table 10 reports the frailty level and the type of care prevalence index for the years from 1995 to 2045. Throughout the acuity levels, our findings predict an important increase in the dependent population. In line with the overall findings from Table 9, we observe serious development in the number of future mildly, moderately and severely dependent persons when compared to 2014. For the year 2045, the index shows that, in the three dependency states, the number of dependent elderly will more than double. This increase is particularly significant for the severely dependent persons. With the year 2014 as baseline, we find an increase by $52 \%$ (factor 1.52) 
in 2025, more than doubling (2.10) in 2035 and close to tripling (2.75) in 2045. We find a similar pattern in the analysis of the mildly and moderately dependent individuals, but of lower scale for the mildly dependent persons.

\begin{tabular}{|c|c|c|c|c|c|c|c|}
\hline Index in year $t$ & 1995 & 2005 & 2014 & 2015 & 2025 & 2035 & 2045 \\
\hline \multicolumn{8}{|l|}{ Frailty level } \\
\hline (1) Mild & 0.13 & 0.18 & 1.00 & 0.95 & 1.24 & 1.64 & 1.98 \\
\hline Moderate & 0.43 & 0.74 & 1.00 & 1.05 & 1.43 & 1.97 & 2.53 \\
\hline (3) Severe & 1.03 & 1.10 & 1.00 & 1.09 & 1.52 & 2.10 & 2.75 \\
\hline \multicolumn{8}{|l|}{ Type of care } \\
\hline (a) At home & - & 0.03 & 1.00 & 0.92 & 1.20 & 1.57 & 1.86 \\
\hline In an inst. & 0.76 & 0.96 & 1.00 & 1.07 & 1.48 & 2.05 & 2.67 \\
\hline
\end{tabular}

In gray shadow: Index $2014=1.00$.

Table 10: Evolution of the dependent population by frailty levels and types of care in the reference population scenario.

Focusing on the prevalence rates by types of care, we see that the number of persons cared for at home closely follows the evolution of mildly dependent persons. The figures related to individuals cared for in an institution are between the moderately and the severely dependent persons. Indeed, the number of persons cared for at home is predicted close to double (1.86) for 2045 while the number of persons receiving care in an institution is to be multiplied by 2.67 . The final evolution will however be strongly influenced by future incentivization schemes that promote, for example, care received at home for mildly dependent elderly (cf. the changes of the law in 2002 and 2011).

\subsection{Discussion on the robustness of the forecasts}

In this part, we discuss the robustness of the forecast among two criteria. First, using the out-of-sample backtest method, we measure the effect of calibrating the model on a short time period only (data from 2011 to 2014). Second, we assess the uncertainty linked to the FSO population projections by comparing the results obtained from the reference scenario with two other projection scenarios, namely the low and the high scenarios (see Table 2 in Section 2.4 for the main characteristics of each scenario).

A natural approach to measure the quality of a prediction model relates to the consideration of an out-of-sample test. This type of backtesting refers to the re-estimation of the model on a smaller population for predicting the behavior of the remaining population and comparing it with its true value. We propose to examine the results obtained from the regression model $\left(4^{\prime}\right)$ by the estimation of the regression model taking into account only the years from 2011 to 2013 (leaving 2014 out). We then predict the prevalence rates for the year 2014 and compare with the 2014's values contained in our dataset. We evaluate the error between the estimate of the dependent population $\hat{\mathcal{N}}_{2014}$ and the observed one $\mathcal{N}_{2014}$ in 2014by using the relative difference $\delta=\left(\hat{\mathcal{N}}_{2014}-\mathcal{N}_{2014}\right) / \mathcal{N}_{2014}$. Table 11 presents the results by cantons, linguistic regions and for the whole country. The reported relative differences $\delta$ show that the predicted number of dependent for the year 2014 is close to the true value in most of the cantons. For example, we observe an absolute relative difference $|\delta|$ below $2 \%$ for fourteen of the twenty-six 
cantons (i.e., AG, AI, BE, BL, BS, GE, GL, LU, SG, SO, SZ, TG, VD and ZH). The accuracy further increases when clustering cantons by linguistic regions. The results display relative differences of $0.22 \%, 0.68 \%$ and $-4.10 \%$ for the regions DE, FR and IT, respectively. Finally, the relative differences for the whole of Switzerland equals $-0.02 \%$ identifying the overall precision of the model. The backtesting illustrates the estimation error in our forecast and highlights the necessary hindsight to have on our predictions.

\begin{tabular}{llllllllll}
\hline \multicolumn{2}{l}{ Cantons } & & & & & & \multicolumn{2}{c}{ Linguistic regions } \\
$\mathrm{AG}$ & -0.67 & $\mathrm{GE}$ & -0.68 & $\mathrm{OW}$ & -4.40 & $\mathrm{UR}$ & -3.54 & $\mathrm{DE}$ & +0.22 \\
$\mathrm{AI}$ & -0.22 & $\mathrm{GL}$ & -0.23 & $\mathrm{SG}$ & -1.28 & $\mathrm{VD}$ & +0.47 & $\mathrm{FR}$ & +0.68 \\
$\mathrm{AR}$ & +3.02 & $\mathrm{GR}$ & +3.02 & $\mathrm{SH}$ & +5.85 & $\mathrm{VS}$ & -5.10 & $\mathrm{IT}$ & -4.10 \\
$\mathrm{BE}$ & +1.65 & $\mathrm{JU}$ & -2.90 & $\mathrm{SO}$ & -1.55 & $\mathrm{ZG}$ & -2.92 & Whole country \\
$\mathrm{BL}$ & +1.00 & $\mathrm{LU}$ & +1.66 & $\mathrm{SZ}$ & +0.55 & $\mathrm{ZH}$ & -0.13 & $\mathrm{CH}$ & -0.02 \\
$\mathrm{BS}$ & +0.04 & $\mathrm{NE}$ & +11.78 & $\mathrm{TG}$ & +0.19 & & & & \\
$\mathrm{FR}$ & +8.77 & $\mathrm{NW}$ & -8.62 & $\mathrm{TI}$ & -4.10 & & & & \\
\hline
\end{tabular}

Table 11: Relative differences $\delta$ in percent.

Our model also requires assumptions about the future old-age population. For the factor $\hat{\mathcal{P}}_{t, A, G, C}$ in Equation (8) we have used until here the FSO reference scenario. These estimates affect the values of our forecasts. In Table 12, we present the evolution of the dependent population for each canton when using the low and the high FSO population scenarios. At the bottom of the table, we show the values by linguistic regions and for the whole country. The reported numbers compare to the values of the dependent population prevalence index $\hat{I}_{t, C}$ laid out in Table 9. For example, on the horizon 2045, the index coming from the low scenario $\left(\hat{I}_{t, C}^{\mathrm{Low}}\right)$ takes the values of 2.68, 1.49, 2.19, 2.29 and 2.18 for the cantons AG, BS, TI, VD and ZH (see Figure 7$)$. It takes the values of $3.00,1.70,2.55,2.62,2.46$ in the case of the high scenario $\left(\hat{I}_{t, C}^{\mathrm{High}}\right)$. For the whole country in 2045 , we observe that $\hat{I}_{t, C}^{\mathrm{Low}}=2.30$ and $\hat{I}_{t, C}^{\mathrm{High}}=2.60$ which compare to the value from the reference scenario $\hat{I}_{t, C}=2.45$. We observe that the range between the low and the high scenario index values is 0.30 in 2045 , i.e. the size of this interval corresponds to about $10 \%$ of the index value in the reference scenario. Thus, using few variables, i.e., the age class, the gender, the canton, the acuity levels and the population forecasts, our approach gives relevant insight on the evolution of the future dependent elderly population in Switzerland.

\section{Conclusion}

This paper adds to the current body of LTC knowledge in Switzerland by improving the understanding of the factors affecting the prevalence rates. In our work, we determine some of the main drivers affecting the evolution of the population aged $65+$ years in need of LTC and propose a prediction over a 30-year horizon for the future needs of care. Our approach relies on log-linear regression methodologies and proposes a distinction between frailty levels and types of care. We distinguish three frailty levels (mild, moderate and severe) and two types of care (at home and in an institution).

Our results indicate that the age, the gender and the canton of residence are significant factors in determining the need of LTC. We statistically find that older persons present higher prevalence rates than others. A similar result appears when comparing men and women, i.e., women experience more important prevalence rates than men. By taking the canton Zurich as 


\begin{tabular}{ccccccccc}
\hline Year $t$ & \multicolumn{2}{c}{2015} & \multicolumn{2}{c}{2025} & \multicolumn{2}{c}{2035} & \multicolumn{2}{c}{2045} \\
& $\hat{I}_{t, C}^{\text {Low }}$ & $\hat{I}_{t, C}^{\text {High }}$ & $\hat{I}_{t, C}^{\text {Low }}$ & $\hat{I}_{t, C}^{\text {High }}$ & $\hat{I}_{t, C}^{\text {Low }}$ & $\hat{I}_{t, C}^{\text {High }}$ & $\hat{I}_{t, C}^{\text {Low }}$ & $\hat{I}_{t, C}^{\text {High }}$ \\
\hline Cantons & & & & & & & & \\
AG & 1.02 & 1.02 & 1.45 & 1.49 & 2.06 & 2.19 & 2.68 & 3.00 \\
AI & 1.03 & 1.04 & 1.41 & 1.44 & 1.87 & 1.98 & 2.32 & 2.56 \\
AR & 1.04 & 1.04 & 1.34 & 1.37 & 1.75 & 1.87 & 2.15 & 2.38 \\
BE & 1.04 & 1.04 & 1.36 & 1.40 & 1.81 & 1.93 & 2.21 & 2.47 \\
BL & 1.04 & 1.04 & 1.39 & 1.43 & 1.79 & 1.90 & 2.11 & 2.36 \\
BS & 1.02 & 1.02 & 1.14 & 1.18 & 1.30 & 1.40 & 1.49 & 1.70 \\
FR & 1.09 & 1.09 & 1.51 & 1.55 & 2.18 & 2.32 & 2.87 & 3.20 \\
GE & 1.03 & 1.03 & 1.35 & 1.41 & 1.72 & 1.88 & 2.05 & 2.40 \\
GL & 1.03 & 1.04 & 1.33 & 1.38 & 1.78 & 1.90 & 2.22 & 2.51 \\
GR & 1.03 & 1.03 & 1.40 & 1.44 & 1.90 & 2.04 & 2.32 & 2.63 \\
JU & 1.01 & 1.02 & 1.31 & 1.37 & 1.72 & 1.85 & 2.12 & 2.39 \\
LU & 1.05 & 1.05 & 1.40 & 1.43 & 1.87 & 1.99 & 2.43 & 2.70 \\
NE & 1.10 & 1.10 & 1.34 & 1.38 & 1.68 & 1.80 & 2.00 & 2.26 \\
NW & 0.98 & 0.98 & 1.50 & 1.55 & 2.13 & 2.29 & 2.55 & 2.89 \\
OW & 1.00 & 1.00 & 1.51 & 1.55 & 2.21 & 2.36 & 2.84 & 3.21 \\
SG & 1.02 & 1.03 & 1.39 & 1.43 & 1.88 & 2.01 & 2.37 & 2.65 \\
SH & 1.07 & 1.07 & 1.42 & 1.46 & 1.86 & 1.98 & 2.29 & 2.57 \\
SO & 1.02 & 1.03 & 1.39 & 1.42 & 1.84 & 1.95 & 2.30 & 2.56 \\
SZ & 1.04 & 1.04 & 1.53 & 1.58 & 2.23 & 2.37 & 2.95 & 3.31 \\
TG & 1.04 & 1.04 & 1.51 & 1.55 & 2.18 & 2.32 & 2.88 & 3.22 \\
TI & 0.99 & 0.99 & 1.37 & 1.44 & 1.81 & 2.00 & 2.19 & 2.55 \\
UR & 0.99 & 0.99 & 1.34 & 1.38 & 1.82 & 1.95 & 2.26 & 2.54 \\
VD & 1.03 & 1.03 & 1.35 & 1.40 & 1.81 & 1.95 & 2.29 & 2.62 \\
VS & 0.98 & 0.99 & 1.41 & 1.46 & 1.98 & 2.14 & 2.51 & 2.85 \\
ZG & 1.02 & 1.02 & 1.51 & 1.55 & 2.11 & 2.26 & 2.66 & 3.02 \\
ZH & 1.03 & 1.03 & 1.35 & 1.39 & 1.77 & 1.90 & 2.18 & 2.46 \\
\hline Linguistic regions & & & & & & \\
DE & 1.03 & 1.03 & 1.38 & 1.42 & 1.84 & 1.97 & 2.30 & 2.58 \\
FR & 1.03 & 1.03 & 1.38 & 1.43 & 1.86 & 2.01 & 2.33 & 2.66 \\
IT & 0.99 & 0.99 & 1.37 & 1.44 & 1.81 & 2.00 & 2.19 & 2.55 \\
\hline Whole & country & & & & & & & \\
CH & 1.03 & 1.03 & 1.38 & 1.42 & 1.85 & 1.98 & 2.30 & 2.60 \\
\hline & & & & & & & &
\end{tabular}

Table 12: Evolution of the dependent population by cantons in the low and the high population scenarios.

baseline, we observe that the prevalence rates also depend on the canton of residence. However, we should mitigate the canton effect because it might be related to some hidden population characteristics that we cannot observe, e.g., cultural aspects linked to help provided by relatives and differences in cantonal procedures. The distinction in two series of models details the age, gender and canton effects on prevalence rates by frailty levels and types of care. We discover that the age and the gender have a larger influence on the prevalence rates for both the severely dependent persons and the ones being cared for in an institution. Our findings also highlight similarities between mildly dependent persons and persons cared for at home. A simi- 
lar conclusion appears when looking at the moderately and the severely dependent persons and individuals receiving care in an institution. This confirms that the major part of the persons in moderate and severe dependency is receiving care in an institution while mildly dependent persons only require home-based care.

Our forecasts imply a serious increase of the demand in LTC over the next thirty years when compared to the number of dependent elderly in 2014. By considering the cantons of residence in our forecasts, we observe three main clusters. The cantons of Bern, Vaud, Ticino, Zurich exhibit for 2045 an increase in the dependent population by a factor close to 2.4 when compared to the year 2014. We observe a more severe trend for the cantons of Aargau, Lucerne, Nidwalden, St.Gallen, Valais and Zug showing a factor of 2.6. The cantons of Basel-Landschaft, Genève, Jura and Neuchâtel present a lower change with an increase in the 2045 dependent population by a factor close to 2.2 in comparison to 2014. Furthermore, we expect the moderately and severely dependent population to double by 2035 and close to triple by 2045 . We see analogous results for the persons cared for in an institution. The situation is slightly less dramatic for both the mildly dependent persons and the ones receiving care at home because the doubling in population is expected by 2045 instead of 2035. Our projections highlight the relevance of the evolution in Switzerland and may hint the needs for action-taking. Certain hindsight is necessary in particular, since our projections rely on population forecasts and are impacted among others by system changes, incentivization schemes (e.g., towards more care at home), medical and technological progress.

Beyond the present study, we see various streams for further research. On a first stage, our study could be improved by analyzing the impact of further sociodemographic factors, e.g., the social class and the nationality on the prevalence rates. By accessing the underlying longitudinal data, further analyses could consider the age at entry and the time spent in the different states of dependence, the probability of different paths and evaluate their effect on the overall dependence (Fuino and Wagner, 2018). Finally, pathologies, functional limitations in ADLs and causes of death are factors affecting the dependence of elderly (Marengoni et al., 2011; Barnett et al., 2012; Biessy, 2016) and their analysis might lead to a better understanding of prevalence rate drivers.

\section{References}

Ai, C. and E. C. Norton, 2008, A Semiparametric Derivative Estimator in Log Transformation Models, The Econometrics Journal, 11(3):538-553.

Barnett, K., S. W. Mercer, M. Norbury, G. Watt, S. Wyke, and B. Guthrie, 2012, Epidemiology of Multimorbidity and Implications for Health Care, Research, and Medical Education: A Cross-Sectional Study, The Lancet, 380(9836):37-43.

Biessy, G., 2016, A Semi-Markov Model with Pathologies for Long-Term Care Insurance, Working Paper, Université d'Évry Val d'Essonne.

Black, M. A. and B. A. Craig, 2002, Estimating Disease Prevalence in the Absence of a Gold Standard, Statistics in Medicine, 21(18):2653-2669.

Bonsang, E., 2009, Does informal care from children to their elderly parents substitute for formal care in Europe?, Journal of Health Economics, 28(1):143-154.

Brown, J., N. Coe, and A. Finkelstein, 2007, Medicaid Crowd-Out of Private Long-Term Care 
Insurance Demand: Evidence from the Health and Retirement Survey, Technical Report August, National Bureau of Economic Research, Cambridge, MA.

Callahan, C. M., G. Arling, W. Tu, M. B. Rosenman, S. R. Counsell, T. E. Stump, and H. C. Hendrie, 2012, Transitions in Care for Older Adults with and Without Dementia, Journal of the American Geriatrics Society, 60(5):813-820.

Colombo, F., 2012, Typology of Public Coverage for Long-Term Care in OECD Countries, In J. Costa-Font and C. Courbage, editors, Financing Long-Term Care in Europe, chapter 2, pages 17-40. Palgrave Macmillan, New York.

Cook, R. D. and S. Weisberg, 1983, Diagnostics for Heteroscedasticity in Regression, Biometrika, 70(1):1-10.

Cosandey, J., 2016, De Nouvelles Mesures pour les Soins des Personnes Agées, Avenir Suisse.

Costa-Font, J., R. Wittenberg, C. Patxot, A. Comas-Herrera, C. Gori, A. di Maio, L. Pickard, A. Pozzi, and H. Rothgang, 2008, Projecting Long-Term Care Expenditure in Four European Union Member States: the Influence of Demographic Scenarios, Social Indicators Research, 86(2):303-321.

Courbage, C., G. Montoliu-Montes, and J. Wagner, 2018, Informal Care, Long Term-Care Insurance and Intra-family Moral Hazard: Empirical Evidence from Southern Europe, Working Paper, University of Lausanne.

Courbage, C. and P. Zweifel, 2011, Two-Sided Intergenerational Moral Hazard, Long-Term Care Insurance, and Nursing Home Use, Journal of Risk and Uncertainty, 43(1):65-80.

Crimmins, E. M., J. K. Kim, and A. Solé-Auró, 2011, Gender Differences in Health: Results from SHARE, ELSA and HRS, European Journal of Public Health, 21(1):81-91.

Czado, C. and F. Rudolph, 2002, Application of Survival Analysis Methods to Long-Term Care Insurance, Insurance: Mathematics and Economics, 31(3):395-413.

De Meijer, C., M. Koopmanschap, T. B. D' Uva, and E. van Doorslaer, 2011, Determinants of Long-Term Care Spending: Age, Time to Death or Disability?, Journal of Health Economics, 30(2):425-438.

Efron, B., 1979, Bootstrap Methods: Another Look at the Jackknife, The Annals of Statistics, $7(1): 1-26$.

Efron, B. and R. J. Tibshirani, 1993, An Introduction to the Bootstrap. Springer US, Boston, MA.

Eggink, E., I. Woittiez, and M. Ras, 2016, Forecasting the Use of Elderly Care: a Static MicroSimulation Model, European Journal of Health Economics, 17(6):681-691.

El Bernoussi, R. and M. Rockinger, 2017, Besoin en Logements pour Personnes Agées en Suisse, Technical Report, Lausanne.

Eugster, B., R. Lalive, A. Steinhauer, and J. Zweimüller, 2011, The Demand for Social Insurance: Does Culture Matter?, The Economic Journal, 121(556):413-448.

European Commission, 2015, The 2015 Ageing Report: Economic and Budgetary Projections for the 28 EU Member States (2013-2060), volume 3/2015.

Federal Assembly, 2017, Loi Fédérale sur l'Assurance-Vieillesse et Survivants (LAVS) 831.10.

Federal Statistical Office, 1998, Deux Siècles d'Histoire Démographique Suisse. Berne.

Federal Statistical Office, 2015, Les Scénarios de l'Evolution de la Population de la Suisse 2015-2045. Neuchâtel.

Federal Statistical Office, 2017, Tables de Mortalité pour la Suisse 2008/2013. Neuchâtel. 
Fong, J. H., 2017, Old-age Frailty Patterns and Implications for Long-term Care Programmes, The Geneva Papers on Risk and Insurance - Issues and Practice, 42(1):114-128.

Fong, J. H., M. Sherris, and J. Yap, 2017, Forecasting Disability: Application of a Frailty Model, Scandinavian Actuarial Journal, 2017(2):125-147.

Fuino, M. and J. Wagner, 2018, Long-Term Care Models and Dependence Probability Tables by Acuity Level: New Empirical Evidence from Switzerland, Insurance: Mathematics and Economics, 81:51-70.

Gentili, E., G. Masiero, and F. Mazzonna, 2017, The Role of Culture in Long-Term Care Arrangement Decisions, Journal of Economic Behavior ES Organization, 143(2017):186-200.

Han, L., H. Allore, T. Murphy, T. Gill, P. Peduzzi, and H. Lin, 2013, Annals of Epidemiology Dynamics of Functional Aging Based on Latent-Class Trajectories of Activities of Daily Living, Annals of Epidemiology, 23(2):87-92.

Karlsson, M., L. Mayhew, R. Plumb, and B. Rickayzen, 2006, Future Costs for Long-Term Care: Cost Projections for Long-Term Care for Older People in the United Kingdom, Health Policy, 75(2):187-213.

Katz, P. R., 2011, An international perspective on long term care: Focus on nursing Homes, Journal of the American Medical Directors Association, 12(7):487-492.

Kwon, H.-S., C.-S. Lee, and J.-S. Hur, 2012, Projecting the Cost of Long-Term Care Insurance in Korea, Asia-Pacific Journal of Risk and Insurance, 7(1):1-20.

Le Corre, P.-Y., 2012, Long-Term Care Insurance: Building a Successful Development, In J. Costa-Font and C. Courbage, editors, Financing Long-Term Care in Europe, chapter 4, pages 53-72. Palgrave Macmillan, London.

Lopes, R., 2011, Kolmogorov-Smirnov Test, In International Encyclopedia of Statistical Science. Springer, Berlin, Heidelberg.

Lumley, T., P. Diehr, S. Emerson, and L. Chen, 2002, The Importance of the Normality Assumption in Large Public Health Data Sets, Annual Reviews of Public Health, 23(1):151-169.

Manning, W. G., 1998, The Logged Dependent Variable, Heteroscedasticity, and the Retransformation Problem, Journal of Health Economics, 17(3):283-295.

Manning, W. G. and J. Mullahy, 2001, Estimating Log models: To Transform or Not to Transform?, Journal of Health Economics, 20(4):461-494.

Marengoni, A., S. Angleman, R. Melis, F. Mangialasche, A. Karp, A. Garmen, B. Meinow, and L. Fratiglioni, 2011, Aging with Multimorbidity: A Systematic Review of the Literature, Ageing Research Reviews, 10(4):430-439.

Mathers, C., 1996, Trends in Health Expectancies in Australia 1981-1993, Journal of the Australian Population Association, 13(1):1-15.

Mathers, C., T. Vos, and C. Stevenson, 2001, The Burden of Disease and Injury in Australia, World Health Organisation, 23(1):1076-1084.

Meguro, K., N. Tanaka, M. Kasai, K. Nakamura, H. Ishikawa, M. Nakatsuka, M. Satoh, and Y. Ouchi, 2012, Prevalence of Dementia and Dementing Diseases in the Old-Old Population in Japan: The Kurihara Project. Implications for Long-Term Care Insurance Data, Psychogeriatrics, 12(4):226-234.

Monod-Zorzi, S., L. Seematter-Bagnoud, C. Büla, S. Pellegrini, and H. Jaccard Ruedin, 2007, Maladies Chroniques et Dépendance Fonctionnelle des Personnes Agées. Observatoire Suisse de la Santé, Neuchâtel.

Nichols, B. L., C. R. Davis, and D. R. Richardson, 2010, An Integrative Review of Global 
Nursing Workforce Issues., Annual Review of Nursing Research, 28:113-32.

OECD, 2015, Health at a Glance 2015. Health at a Glance. OECD Publishing, Paris.

Ohri, A., 2012, $R$ for Business Analytics. Springer, New York.

Pauly, M. V., 1990, The Rational Nonpurchase of Long-Term-Care Insurance, Journal of Political Economy, 98(1):153-168.

Rockinger, M. and J. Wagner, 2016, Les Soins et la Dépendance: Un Risque Systémique, Le Temps, 28 June.

Schünemann, J., H. Strulik, and T. Trimborn, 2017, The Gender Gap in Mortality: How Much is Explained by Behavior?, Journal of Health Economics, 54:79-90.

Stock, J. H. and M. M. Watson, 2012, Introduction to Econometrics. Pearson Education Limited, Harlow, 3rd edition.

Swiss Re, 2014, How Will We Care? Finding Sustainable Long-Term Care Solutions for an Ageing World, Sigma, No 5/2014.

Trevor, B. and P. Adrian, 1979, A Simple Test for Heteroscedasticity and Random Coefficient Variation, Econometrica, 47(5):1287-1294.

United Nations, 2016, Human Development Report 2016. United Nations Development Programme, New York.

Weaver, F., 2012, Long-Term Care Financing in Switzerland, In J. Costa-Font and C. Courbage, editors, Financing Long-Term Care in Europe, chapter 15, pages 279-299. Palgrave Macmillan, New York.

Wooldridge, J. M., 2013, Introductory Econometrics: A Modern Approach. South-Western Cengage Learning, 5th edition.

World Health Organization, 2014, Migration of Health Workers. WHO Document Production Services, Geneva.

Xue, Q.-l., 2011, The Frailty Syndrome: Definition and Natural History, Clinics in Geriatric Medicine, 27(1):1-15.

Yip, A. G., C. Brayne, F. E. Matthews, and MRC Cognitive Function and Ageing Study, 2006, Risk Factors for Incident Dementia in England and Wales: The Medical Research Council Cognitive Function and Ageing Study. A Population-Based Nested Case-Control Study., Age and Ageing, 35(2):154-60.

Zweifel, P., S. Felder, and A. Werblow, 2004, Population Ageing and Health Care Expenditure: New Evidence on the Red Herring, The Geneva Papers on Risk and Insurance - Issues and Practice, 29(4):652-666. 